\title{
BOUNDARY NULL-CONTROLLABILITY OF TWO COUPLED PARABOLIC EQUATIONS: SIMULTANEOUS CONDENSATION OF EIGENVALUES AND EIGENFUNCTIONS*
}

\author{
EL HADJI SAMB**
}

\begin{abstract}
Let the matrix operator $L=D \partial_{x x}+q(x) A_{0}$, with $D=\operatorname{diag}(1, \nu), \nu \neq 1, q \in L^{\infty}(0, \pi)$, and $A_{0}$ is a Jordan block of order 1 . We analyze the boundary null controllability for the system $y_{t}-L y=0$. When $\sqrt{\nu} \notin \mathbb{Q}_{+}^{*}$ and $q$ is constant, $q=1$ for instance, there exists a family of root vectors of $\left(L^{*}, \mathcal{D}\left(L^{*}\right)\right)$ forming a Riesz basis of $L^{2}\left(0, \pi ; \mathbb{R}^{2}\right)$. Moreover F. Ammar Khodja et al. [J. Funct. Anal. 267 (2014) 2077-2151] shows the existence of a minimal time of control depending on condensation of eigenvalues of $\left(L^{*}, \mathcal{D}\left(L^{*}\right)\right)$, that is to say the existence of $T_{0}(\nu)$ such that the system is null controllable at time $T>T_{0}(\nu)$ and not null controllable at time $T<T_{0}(\nu)$. In the same paper, the authors prove that for all $\tau \in[0,+\infty]$, there exists $\nu \in] 0,+\infty\left[\operatorname{such}\right.$ that $T_{0}(\nu)=\tau$. When $q$ depends on $x$, the property of Riesz basis is no more guaranteed. This leads to a new phenomena: simultaneous condensation of eigenvalues and eigenfunctions. This condensation affects the time of null controllability.
\end{abstract}

Mathematics Subject Classification. 93B05, 93C20, 93C25, 30E05, 35K90, 35P10.

Received February 6, 2019. Accepted November 30, 2020.

\section{INTRODUCTION AND MAIN RESULTS}

This paper deals with the controllability of two coupled one-dimensional parabolic equations, with different diffusion coefficients, where the control is exerted at one boundary point. Let us fix $T>0$ and consider the following control problem:

$$
\begin{cases}y_{t}+L y=0 & \text { in } Q_{T}:=(0, \pi) \times(0, T), \\ y(0, \cdot)=B u, \quad y(\pi, \cdot)=0 & \text { on }(0, T), \\ y(\cdot, 0)=y_{0} & \text { in }(0, \pi),\end{cases}
$$

where

$$
L=-D \partial_{x x}+q(x) A_{0}, D=\operatorname{diag}(1, \nu), \text { with } \nu>0, A_{0}=\left(\begin{array}{cc}
0 & 1 \\
0 & 0
\end{array}\right) \text { and } B=\left(\begin{array}{c}
0 \\
1
\end{array}\right)
$$

${ }^{*}$ The author thanks A. Benabdallah, F. Boyer et M. Morancey for useful conversations. I am very grateful to them.

Keywords and phrases: Control theory; parabolic partial differential equations; minimal null control time.

Aix Marseille Université, CNRS, Centrale Marseille, I2M, UMR 7373, 13453 Marseille, France.

** Corresponding author: sambgoree@gmail.com 
$q \in L^{\infty}(0, \pi)$ is a given function, $y_{0}$ is the initial datum and $u \in L^{2}(0, T)$ is the control function. Equivalently, the previous system (1.1) can be written as

$$
\begin{cases}\partial_{t} y_{1}=\partial_{x x} y_{1}-q y_{2} & \text { in } Q_{T}:=(0, \pi) \times(0, T), \\ \partial_{t} y_{2}=\nu \partial_{x x} y_{2} & \text { in } Q_{T}, \\ y_{1}(0, \cdot)=0, \quad y_{2}(0, \cdot)=u, \quad y(\pi, \cdot)=0 & \text { on }(0, T), \\ y(\cdot, 0)=y_{0} & \text { in }(0, \pi) .\end{cases}
$$

It is known (see [13], Prop. 2.2) that for any given initial data $y_{0} \in H^{-1}\left(0, \pi ; \mathbb{R}^{2}\right)$ and $u \in L^{2}(0, T)$, system (1.1) possesses a unique solution defined by transposition which satisfies

$$
y \in L^{2}\left(Q_{T} ; \mathbb{R}^{2}\right) \cap C^{0}\left([0, T] ; H^{-1}\left(0, \pi ; \mathbb{R}^{2}\right)\right)
$$

and depends continuously on the data $u$ and $y_{0}$, i.e., there exists a constant $C=C(T)>0$ such that

$$
\|y\|_{L^{2}\left(Q_{T} ; \mathbb{R}^{2}\right)}+\|y\|_{C^{0}\left([0, T] ; H^{-1}\left(0, \pi ; \mathbb{R}^{2}\right)\right)} \leq C\left(\left\|y_{0}\right\|_{H^{-1}\left(0, \pi ; \mathbb{R}^{2}\right)}+\|u\|_{L^{2}(0, T)}\right) .
$$

Let us introduce the notion of null and approximate controllability for this kind of systems.

1. System (1.1) is approximately controllable in $H^{-1}\left(0, \pi ; \mathbb{R}^{2}\right)$ at time $T$ if for every $y_{0}, y_{d} \in H^{-1}\left(0, \pi ; \mathbb{R}^{2}\right)$ and for every $\varepsilon>0$, there exists a control $u \in L^{2}(0, T)$ such that the solution to (1.1) associated to $y_{0}$ and $u$ satisfies

$$
\left\|y(\cdot, T)-y_{d}\right\|_{H^{-1}\left(0, \pi ; \mathbb{R}^{2}\right)} \leq \varepsilon .
$$

2. System (1.1) is null controllable at time $T$ if for every initial condition $y_{0} \in H^{-1}\left(0, \pi ; \mathbb{R}^{2}\right)$, there exists a control $u \in L^{2}(0, T)$ such that the solution $y$ to (1.1) satisfies

$$
y(\cdot, T)=0 \quad \text { in } H^{-1}\left(0, \pi ; \mathbb{R}^{2}\right) .
$$

The null controllability of parabolic partial differential equations has been widely studied since the pioneering work of [12]. From the works of $[14,16]$, it was commonly admitted that, in the context of parabolic partial differential equations, there is no restriction on the final time $T$. But recently the study of particular examples highlighted the existence of a positive minimal time $T_{0}$ for null controllability. Actually, such an example was already provided in the 70s in [8]. The more recent results concerning such strictly positive a minimal time have been obtained in contexts of control of coupled parabolic equations $[2,3,9]$ and more generally see [4].

The main goal of this article is to address a new phenomenon arising in the null controllability issue for system (1.1). Let us first recall some known results about the controllability properties of scalar parabolic systems. The null controllability problem for scalar parabolic systems has been first considered in the onedimensional case. In [11, 12], using the moment method, H. O. Fattorini and D. L. Russell gave a positive answer for null controllability problem of considered parabolic system. Also, the authors proved a general result on existence of a bi-orthogonal family to $\left\{e^{-\Lambda_{k} t}\right\}_{k \geq 1}$ in $L^{2}(0, T)$ which fulfils appropriate bounds if the sequence $\Lambda_{k} \subset \mathbb{R}_{+}$satisfies the following gap property:

$$
\sum_{k \geq 1} \frac{1}{\Lambda_{k}}<\infty \quad \text { and } \quad\left|\Lambda_{k}-\Lambda_{l}\right| \geq \rho|k-l|, \quad \forall k, l \geq 1,
$$


for a constant $\rho>0$. In 1973, S. Dolecki addressed the pointwise controllability at time $T$ of the one-dimensional heat equation (see [8]). S. Dolecki exhibited a minimal time which depends on the point $x_{0}$. To our knowledge, this was the first result on null-controllability of parabolic problems where a minimal time of control appears. System (1.1) is a particular class of more general $n \times n$ parabolic control systems of the form:

$$
\begin{cases}\partial_{t} y-\left(D \partial_{x x}+A(x)\right) y=0 & \text { in } Q_{T}:=(0, \pi) \times(0, T) \\ y(0, \cdot)=B u, \quad y(\pi, \cdot)=0 & \text { on }(0, T), \\ y(\cdot, 0)=y_{0} & \text { in }(0, \pi) .\end{cases}
$$

Here, $D=\operatorname{diag}\left(d_{1}, . ., d_{n}\right)$, with $d_{i}>0$ for $i: 1 \leq i \leq n, A=\left(a_{i j}\right)_{1 \leq i \leq n} \in L^{\infty}\left((0, \pi) ; M_{n}(\mathbb{R})\right)$ and $B \in$ $\mathcal{L}\left(\mathbb{R}^{m}, \mathbb{R}^{n}\right)$. In system $(1.5), u \in L^{2}\left(0, T ; \mathbb{R}^{m}\right)$ is the control and we want to control the complete system $(n$ equations) by means of $m$ controls exerted on the boundary condition at point $x=0$. Observe that the most interesting (and difficult) case is the case $m<n$.

The first results of null controllability for system (1.5) was obtained in [13] in the case $n=2, m=1$ and $D=I d, A \in M_{n}(\mathbb{R})$. This result was generalized by [1] to the case $n \geq 2, m \geq 1$. In these two papers, the authors used the method of moments of Fattorini-Russell to give a necessary and sufficient condition of null controllability at any time $T>0$ for system (1.5). In both cases, the sequence of eigenvalues $\Lambda=\left\{\Lambda_{k}\right\}_{k \geq 1} \subset \mathbb{R}_{+}$ of the matrix operator $\mathcal{A}=I d \partial_{x x}+A$ with Dirichlet boundary conditions continue to satisfy the $g a p$ condition in (1.4). As in the scalar case, this gap property (together with appropriate properties for the coupling and control matrices $A$ and $B$ ) provides the null controllability result for system (1.5) at any positive time.

In [2], the authors are interested in the extension of the previous null controllability results for system (1.5) to the case where $D \neq I d, A \in M_{n}(\mathbb{R}), n>1$ and $m<n$. The main difference with the case $D=I d$ lies in the behavior of the sequence of eigenvalues of the matrix operator $\mathcal{A}:=D \partial_{x x}+A$. The operator $-\mathcal{A}$ admits a sequence of eigenvalues $\Lambda=\left\{\Lambda_{k}\right\}_{k \geq 1}$ which does not satisfy the gap condition appearing in (1.4) but the operator $-\mathcal{A}$ is diagonalizable, i.e., its eigenfunctions form a Riesz basis. As a consequence, the authors show the existence of a minimal time of control $T_{0} \in[0,+\infty]$, depending to the so-called condensation index, $c(\Lambda)$, of the sequence $\Lambda$ of eigenvalues of the operator $-\mathcal{A}$. To our knowledge, this condensation index has been introduced for the first time by V. Bernstein (see [6]) for increasing real sequences and later extended by J. R. Shackell (see [19]) to complex sequences. Roughly speaking, if we consider the complex sequence $\Lambda=\left\{\Lambda_{k}\right\}_{k \geq 1} \subset \mathbb{C}$, the condensation index of $\Lambda$, is a measure of the way how $\Lambda_{n}$ approaches $\Lambda_{m}$ for $n \neq m$.

In [3], the authors consider the case where $D=I d, A(x)=q(x) A_{0}$, with $q \in L^{\infty}(0, \pi), n=2$ and $m=1$. In this case the operator $L^{*}:=-I d \partial_{x x}+q(x) A_{0}^{*}$ admits a sequence of eigenvalues $\Lambda=\left\{\Lambda_{k}\right\}_{k \geq 1}$ which satisfy the gap condition appearing in (1.4) moreover using the eigenfunctions and the generalized eigenfunctions of the operator $L^{*}$, we can construct a Riesz basis for the space $L^{2}\left(0, \pi ; \mathbb{R}^{2}\right)$. As a consequence, the authors show the existence of a minimal time of control $T_{0}(q)$. They also proved that for any $\tau \in[0,+\infty]$, there exists $q \in L^{\infty}(0, \pi)$ such that $T_{0}(q)=\tau$.

In this paper, we study the null controllability properties of system (1.5) to the case where $D \neq I d$ and $A(x)=q(x) A_{0}, q \in L^{\infty}(0, \pi), n=2$ and $m=1$. This situation may seem like a simple perturbation of previous cases (in $[2,3]$ ). It is not true, it contains a new phenomenon: simultaneous condensation of eigenvalues and eigenfunctions. This phenomenon was excluded from all previous cases because of the property that the family of eigenfunctions of the operator $\left(L^{*}, \mathcal{D}\left(L^{*}\right)\right)$ form a Riesz basis for the Hilbert space where the system is posed. This condensation of eigenfunctions can compensate the condensation of eigenvalues and the minimal time of control is affected. In [2] as in [3] the authors proved the controllability by using the usual moments method but this method does not take into account such phenomena of eigenfunctions condensation. Under appropriate assumptions on $D$ and $q \in L^{\infty}(0, \pi)$, the operator $L^{*}=-D \partial_{x x}+q(x) A_{0}^{*}$ admits a sequence of eigenvalues $\Lambda=\left\{\Lambda_{k}\right\}_{k \geq 1}$ which does not satisfy the gap condition appearing in (1.4) however the sequence of associated eigenfunctions is complete but it is not a Riesz basis (see Prop. 2.4) for some $\sqrt{\nu} \notin \mathbb{Q}_{+}^{*}$ and $q \in L^{\infty}(0, \pi)$. As a consequence, we will see that a minimal time of control $T_{0} \in[0,+\infty]$, depends simultaneously of condensation 
of eigenvalues and associated eigenfunctions of $\left(L^{*}, \mathcal{D}\left(L^{*}\right)\right)$. To this end, we will use the block methods moment developed by A. Benabdallah, F. Boyer, M. Morancey (in [5]).

The plan of the paper is the following one: In Section 1, we address some known results about the controllability of parabolic system and we give the main result of this work. In Section 2 we study the null-controllability of system (1.1) when $\sqrt{\nu} \notin \mathbb{Q}_{+}^{*}, \sqrt{\nu}>1$ (resp. $\sqrt{\nu}<1$ ). Section 3 is devoted to null-controllability of system (1.1) when $\sqrt{\nu} \in \mathbb{Q}_{+}^{*}$. Finally, in the Appendix we give additional properties of our main result.

Let us present our first boundary control results, when $\sqrt{\nu} \notin \mathbb{Q}_{+}^{*}$. Let us first introduce some notations. Let $B, A_{0}$ and $D$ given by $(1.2)$ and a function $q \in L^{\infty}(0, \pi)$. Let us consider the operator

$$
L^{*}:=-D \partial_{x x}+q(x) A_{0}^{*}: \mathcal{D}\left(L^{*}\right) \subset L^{2}\left(0, \pi ; \mathbb{R}^{2}\right) \longrightarrow L^{2}\left(0, \pi ; \mathbb{R}^{2}\right)
$$

with domain $\mathcal{D}\left(L^{*}\right)=H^{2}\left(0, \pi ; \mathbb{R}^{2}\right) \cap H_{0}^{1}\left(0, \pi ; \mathbb{R}^{2}\right)$. Given $k \geq 1$, let us consider

$$
\Phi_{1, k}^{*}:=\left(\begin{array}{c}
\varphi_{k} \\
\psi_{k}
\end{array}\right), \Phi_{2, k}^{*}:=\left(\begin{array}{l}
0 \\
\varphi_{k}
\end{array}\right)
$$

where $\psi_{k}$ is the unique solution of problem:

$$
\left\{\begin{array}{l}
\nu \psi_{k}^{\prime \prime}+k^{2} \psi_{k}=q \varphi_{k} \quad \text { in }(0, \pi) \\
\psi(0)=\psi(\pi)=0
\end{array}\right.
$$

Assume that $B^{*} D \frac{\partial \Phi_{1, k}^{*}}{\partial x}(0) \neq 0$, for all $k \geq 1$. Let us defined, for any $k \in \mathbb{N}^{*}, i_{k}$ (resp. $j_{k}$ ) as the nearest integer to $\sqrt{\nu} k$ (resp. $\frac{k}{\sqrt{\nu}}$ ), i.e., $\left|\sqrt{\nu} k-i_{k}\right|<\frac{1}{2}$ (resp. $\left|\frac{k}{\sqrt{\nu}}-j_{k}\right|<\frac{1}{2}$ ). Let us denote

$$
\psi_{i, k}:=\frac{\Phi_{i, k}^{*}}{B^{*} D \frac{\partial \Phi_{i, k}^{*}}{\partial x}(0)}, \forall k \geq 1, \quad \forall i=1,2
$$

In the same way, denote

$$
\psi_{1, i_{k}}:=\frac{\Phi_{1, i_{k}}^{*}}{B^{*} D \frac{\partial \Phi_{1, i_{k}}^{*}}{\partial x}(0)} \text { and } \psi_{2, j_{k}}:=\frac{\Phi_{2, j_{k}}^{*}}{B^{*} D \frac{\partial \Phi_{2, j_{k}}^{*}}{\partial x}(0)}, \quad \forall k \geq 1
$$

One has

Theorem 1.1. Suppose $\sqrt{\nu} \notin \mathbb{Q}_{+}^{*}$.

1. The spectrum of $\left(L^{*}, \mathcal{D}\left(L^{*}\right)\right)$ is given by $\sigma\left(L^{*}\right)=\left\{k^{2}\right\}_{k \geq 1} \cup\left\{\nu k^{2}\right\}_{k \geq 1}$ and the corresponding family of eigenfunctions, $\left\{\Phi_{1, k}^{*}, \Phi_{2, k}^{*}\right\}_{k>1}$, is complete in $L^{2}\left(0, \pi ; \mathbb{R}^{2}\right)$.

2. System (1.1) is approximately controllable at time $T>0$, if and only if

$$
B^{*} D \frac{\partial \Phi_{1, k}^{*}}{\partial x}(0) \neq 0, \forall k \geq 1
$$


3. Introduce

$$
\widetilde{T}_{0}:=\max \left\{\limsup _{k \rightarrow+\infty} \frac{\log \left\|\psi_{1, k}\right\|_{H_{0}^{1}}}{k^{2}}, \limsup _{k \rightarrow+\infty} \frac{\log \frac{\left\|\psi_{1, i_{k}}-\psi_{2, k}\right\|_{H_{0}^{1}}}{\left|i_{k}^{2}-\nu k^{2}\right|}}{\nu k^{2}}\right\}=\max \left\{T_{1}, \widetilde{T}_{2}\right\} \in[0,+\infty],
$$

and

$$
\widehat{T}_{0}:=\max \left\{\limsup _{k \rightarrow+\infty} \frac{\log \left\|\psi_{1, k}\right\|_{H_{0}^{1}}}{k^{2}}, \limsup _{k \rightarrow+\infty} \frac{\log \frac{\left\|\psi_{1, k}-\psi_{2, j_{k}}\right\|_{H_{0}^{1}}}{\left|k^{2}-\nu j_{k}^{2}\right|}}{k^{2}}\right\}=\max \left\{T_{1}, \widehat{T}_{2}\right\} \in[0,+\infty] .
$$

(a) If $\sqrt{\nu}>1$ then: system (1.1) is null-controllable in $H^{-1}\left(0, \pi ; \mathbb{R}^{2}\right)$ for $T>\widetilde{T}_{0}$. On the other hand, if $\widetilde{T}_{0}>0$, system (1.1) is not null-controllable in $H^{-1}\left(0, \pi ; \mathbb{R}^{2}\right)$ for $T<\widetilde{T}_{0}$.

(b) If $\sqrt{\nu}<1$ then: system (1.1) is null-controllable in $H^{-1}\left(0, \pi ; \mathbb{R}^{2}\right)$ for $T>\widehat{T}_{0}$. On the other hand, if $\widehat{T}_{0}>0$, system (1.1) is not null-controllable in $H^{-1}\left(0, \pi ; \mathbb{R}^{2}\right)$ for $T<\widehat{T}_{0}$.

Remark 1.2. 1. In Appendix A, we will write in a simple form the expressions of Theorem 1.1 and show that, $\widetilde{T}_{0}$ and $\widehat{T}_{0}$ strongly depends on diffusion coefficient $\sqrt{\nu} \notin \mathbb{Q}_{+}^{*}$ and the coupling function $q \in L^{\infty}(0, \pi)$. Moreover we will remark that $\widehat{T}_{0}=\widehat{T}_{2} \geq T_{1}$ and $\widehat{T}_{2} \geq \widetilde{T}_{2}$ (see (1.12) and (1.13)).

2. Condition (1.11) characterizes the approximate controllability property of system (1.1). Thus, (1.11) is a necessary condition for the null controllability at time $T>0$ of this system.

3. The approximate controllability result stated in Theorem 1.1 does not depend on the final time $T$ : approximate controllability of system (1.1) at a time $T_{0}>0$ is equivalent to the approximate controllability of system at any time $T>0$.

4. We will prove, in Proposition A.2, that $\widetilde{T}_{0}$ (resp. $\widehat{T}_{0}$ ) could take any value on the interval $[0, \infty]$. Thus, when $\widetilde{T}_{0}, \widehat{T}_{0} \in(0, \infty]$, from Theorem 1.1 we deduce that system (1.1) could have the approximate controllability property at a positive time $T$ without being null controllable at this time $T$.

Let us now present our second boundary control results when $\sqrt{\nu}=\frac{i_{0}}{j_{0}} \in \mathbb{Q}_{+}^{*}$, where $i_{0}$ and $j_{0}$ are co-prime $\left(i_{0} \wedge j_{0}=1\right)$.

Theorem 1.3. Let us consider $\sqrt{\nu} \in \mathbb{Q}_{+}^{*}$. Let $B, A_{0}$ and $D$ given by (1.2). To the function $q \in L^{\infty}(0, \pi)$ and $\nu$, we associate the sequence $\left\{I_{k}\right\}_{k \geq 1}$, defined by

$$
I_{k}(\nu, q)=\int_{0}^{\pi} q(s) \varphi_{k}(s) \sqrt{\frac{\pi}{2}} \sin \left(\frac{k}{\sqrt{\nu}}(\pi-s)\right) \mathrm{d} s
$$

Then, one has:

1. System (1.1) is approximately controllable at time $T>0$, if and only if

$$
I_{k}(\nu, q) \neq 0, \quad \forall k \geq 1
$$

2. Assume (1.15) holds and define

$$
T_{0}(\nu, q):=\limsup _{k \rightarrow+\infty} \frac{-\log \left|I_{k}(\nu, q)\right|}{k^{2}} \in[0,+\infty] .
$$


Then if $T>T_{0}(\nu, q)$ system (1.1) is null-controllable at time $T$. On the other hand, if $T_{0}(\nu, q)>0$, system (1.1) is not null-controllable at time $T$ in $H^{-1}\left(0, \pi ; \mathbb{R}^{2}\right)$ for $T<T_{0}(\nu, q)$.

Remark 1.4. Under condition (1.15), the minimal time $T_{0}(\nu, q)$ is well-defined. Moreover, the sequence $\left\{I_{k}(\nu, q)\right\}_{k \geq 1}$ is bounded and $T_{0}(\nu, q) \in[0,+\infty]$.

Let $q \in L^{\infty}(0, \pi)$ and $A_{0}$ given by (1.2). We introduce the backward adjoint problem associated with system (1.1):

$$
\begin{cases}-\theta_{t}-\left(D \partial_{x x}-q A_{0}^{*}\right) \theta=0 & \text { in } Q_{T}, \\ \theta(0, \cdot)=\theta(\pi, \cdot)=0 & \text { on }(0, T), \\ \theta(\cdot, T)=\theta_{0} & \text { in }(0, \pi),\end{cases}
$$

where $\theta_{0} \in L^{2}\left(0, \pi ; \mathbb{R}^{2}\right)$ is a given initial datum. Let us first see that this problem is well posed. One has:

Proposition 1.5. Assume that $\theta_{0} \in L^{2}\left(0, \pi ; \mathbb{R}^{2}\right)$ is given. Then, system (1.17) admits a unique solution $\theta \in$ $L^{2}\left(0, T ; H_{0}^{1}\left(0, \pi ; \mathbb{R}^{2}\right)\right) \cap C^{0}\left([0, T] ; L^{2}\left(0, \pi ; \mathbb{R}^{2}\right)\right)$ and in addition satisfies

$$
\|\theta\|_{L^{2}\left(0, T ; H_{0}^{1}\left(0, \pi ; \mathbb{R}^{2}\right)\right)}+\|\theta\|_{C^{0}\left([0, T] ; L^{2}\left(0, \pi ; \mathbb{R}^{2}\right)\right)} \leq C(T)\left\|\theta_{0}\right\|_{L^{2}\left(0, \pi ; \mathbb{R}^{2}\right)},
$$

for a positive constant $C(T)>0$ independent of $\theta_{0}$. Furthermore, if $\theta_{0} \in H_{0}^{1}\left(0, \pi ; \mathbb{R}^{2}\right)$, then the solution $\theta$ of the adjoint problem (1.17) satisfies

$$
\theta \in L^{2}\left(0, T ; H^{2}\left(0, \pi ; \mathbb{R}^{2}\right) \cap H_{0}^{1}\left(0, \pi ; \mathbb{R}^{2}\right)\right) \cap C^{0}\left([0, T] ; H_{0}^{1}\left(0, \pi ; \mathbb{R}^{2}\right)\right)
$$

and, for a positive constant $C(T)>0$

$$
\|\theta\|_{L^{2}\left(0, T ; H^{2}\left(0, \pi ; \mathbb{R}^{2}\right) \cap H_{0}^{1}\left(0, \pi ; \mathbb{R}^{2}\right)\right)}+\|\theta\|_{C^{0}\left([0, T] ; H_{0}^{1}\left(0, \pi ; \mathbb{R}^{2}\right)\right)} \leq C(T)\left\|\theta_{0}\right\|_{H_{0}^{1}\left(0, \pi ; \mathbb{R}^{2}\right)}
$$

The next proposition provides a relation between systems (1.1) and (1.17):

Proposition 1.6. Let us consider $A_{0}$ and $B$ given by (1.2) and $q \in L^{\infty}(0, \pi)$. Then, for any $y_{0} \in H^{-1}\left(0, \pi ; \mathbb{R}^{2}\right)$, $u \in L^{2}(0, T)$ and $\theta_{0} \in H_{0}^{1}\left(0, \pi ; \mathbb{R}^{2}\right)$, one has

$$
\int_{0}^{T} u(t) B^{*} D \theta_{x}(0, t) \mathrm{d} t=\left\langle y(\cdot, T), \theta_{0}\right\rangle_{H^{-1}, H_{0}^{1}}-\left\langle y_{0}, \theta(\cdot, 0)\right\rangle_{H^{-1}, H_{0}^{1}}
$$

where $y \in L^{2}\left(Q_{T} ; \mathbb{R}^{2}\right) \cap C^{0}\left([0, T] ; H^{-1}\left(0, \pi ; \mathbb{R}^{2}\right)\right)$ and

$$
\theta \in L^{2}\left(0, T ; H^{2}\left(0, \pi ; \mathbb{R}^{2}\right) \cap H_{0}^{1}\left(0, \pi ; \mathbb{R}^{2}\right)\right) \cap C^{0}\left([0, T] ; H_{0}^{1}\left(0, \pi ; \mathbb{R}^{2}\right)\right)
$$

are, resp., the solution to (1.1) and (1.17) associated with $\left(u, y_{0}\right)$ and $\theta_{0}$.

For a proof of the previous results see for instance [20] or [13].

The controllability of system (1.1) can be characterized in terms of appropriate properties of the solutions to the adjoint problem (1.17). More precisely, we have

Proposition 1.7. The following properties are equivalent: 
1. There exists a positive constant $C>0$ such that, for any $y_{0} \in H^{-1}\left(0, \pi ; \mathbb{R}^{2}\right)$, there exists a control $u \in$ $L^{2}(0, T)$ such that

$$
\|u\|_{L^{2}(0, T)} \leq C\left\|y_{0}\right\|_{H^{-1}\left(0, \pi ; \mathbb{R}^{2}\right)}
$$

and the associated state satisfies

$$
y(\cdot, T)=0 \quad \text { in } H^{-1}\left(0, \pi ; \mathbb{R}^{2}\right)
$$

2. There exists a positive constant $C$ such that the observability inequality

$$
\|\theta(\cdot, 0)\|_{H_{0}^{1}\left(0, \pi ; \mathbb{R}^{2}\right)}^{2} \leq C \int_{0}^{T}\left|B^{*} D \theta_{x}(0, t)\right|^{2} \mathrm{~d} t
$$

holds for every $\theta_{0} \in H_{0}^{1}\left(0, \pi ; \mathbb{R}^{2}\right)$. In (1.18), $\theta$ is the adjoint state associated with $\theta_{0}$.

This result is well known, the "Hilbert Uniqueness Method", see [15].

\section{Approximate AND NULL-CONTROLlability ReSUlt With AN IRRATIONAL DIFFUSION COEFFICIENT}

\subsection{Some preliminary results}

Let us consider the vectorial operator

$$
L:=-D \partial_{x x}+q(x) A_{0}: \mathcal{D}(L) \subset L^{2}\left(0, \pi ; \mathbb{R}^{2}\right) \longrightarrow L^{2}\left(0, \pi ; \mathbb{R}^{2}\right)
$$

with domain $\mathcal{D}(L)=H^{2}\left(0, \pi ; \mathbb{R}^{2}\right) \cap H_{0}^{1}\left(0, \pi ; \mathbb{R}^{2}\right)$ and also its adjoint $L^{*}$ given by (1.6).

Proposition 2.1. Let $A_{0}$ be given by (1.2) and consider the operator L given by (1.6) and also denote its adjoint $L^{*}$. Assume that $\sqrt{\nu} \notin \mathbb{Q}$, then,

1. The spectrum of $L^{*}$ is given by $\sigma\left(L^{*}\right)=\left\{k^{2}\right\}_{k \geq 1} \cup\left\{\nu k^{2}\right\}_{k \geq 1}$.

2. Given $k \geq 1$, if

$$
\Phi_{1, k}^{*}:=\left(\begin{array}{c}
\varphi_{k} \\
\psi_{k}
\end{array}\right), \Phi_{2, k}^{*}:=\left(\begin{array}{l}
0 \\
\varphi_{k}
\end{array}\right)
$$

where $\psi_{k}$ is the unique solution of the following problem:

$$
\left\{\begin{array}{l}
\nu \psi_{k}^{\prime \prime}+k^{2} \psi_{k}=q \varphi_{k} \quad \text { in }(0, \pi) \\
\psi_{k}(0)=\psi_{k}(\pi)=0
\end{array}\right.
$$

then

$$
\left(L^{*}-k^{2} I_{d}\right) \Phi_{1, k}^{*}=0 \quad \text { and } \quad\left(L^{*}-\nu k^{2} I_{d}\right) \Phi_{2, k}^{*}=0 .
$$


Moreover, an explicit expression of $\psi_{k}$ is given by:

$$
\psi_{k}(x)=\psi_{k}^{\prime}(0) \frac{\sqrt{\nu}}{k} \sin \left(\frac{k x}{\sqrt{\nu}}\right)+\frac{\sqrt{\nu}}{\nu k} \int_{0}^{x} \sin \left(\frac{k}{\sqrt{\nu}}(x-\xi)\right) q(\xi) \varphi_{k}(\xi) \mathrm{d} \xi,
$$

where

$$
\psi_{k}^{\prime}(0)=-\frac{\int_{0}^{\pi} q(s) \varphi_{k}(s) \sin \left(\frac{k}{\sqrt{\nu}}(\pi-s)\right) \mathrm{d} s}{\nu \sin \left(\frac{k \pi}{\sqrt{\nu}}\right)}
$$

Remark 2.2. Consider the following problem

$$
\left\{\begin{array}{l}
y^{\prime \prime}+\lambda y=f(x) \quad \text { in }(0, \pi) \\
y(0)=0
\end{array}\right.
$$

where $\lambda \in \mathbb{R}_{+}^{*}$. The general solution to (2.3) is given by

$$
y(x)=a \frac{\sin (\sqrt{\lambda} x)}{\sqrt{\lambda}}+\frac{1}{\sqrt{\lambda}} \int_{0}^{x} \sin (\sqrt{\lambda}(x-s)) f(s) \mathrm{d} s, \quad a \in \mathbb{R}^{*},
$$

and

$$
y^{\prime}(x)=a \cos (\sqrt{\lambda} x)+\int_{0}^{x} \cos (\sqrt{\lambda}(x-s)) f(s) \mathrm{d} s
$$

consequently

$$
y^{\prime}(0)=a=-\frac{\int_{0}^{\pi} f(s) \sin (\sqrt{\lambda}(\pi-s)) \mathrm{d} s}{\sin (\sqrt{\lambda} \pi)}, \quad \text { if } \quad \sqrt{\lambda} \notin \mathbb{N}^{*}
$$

On the other hand, for all $\sqrt{\lambda} \notin \mathbb{N}^{*}$ :

$$
y(x)=\sum_{n \geq 1} \frac{\int_{0}^{\pi} f(x) \varphi_{n}(x) \mathrm{d} x}{\left(\lambda-n^{2}\right)} \varphi_{n}(x) .
$$

\section{Proof of Proposition 2.1.}

Let us assume $\sqrt{\nu} \notin \mathbb{Q}$. Let $\lambda$ be an eigenvalue of $L^{*}$ and $y=\left(y_{1}, y_{2}\right)^{T}$ an associated eigenfunction. Thus $y$ is a solution of the following problem:

$$
\left\{\begin{array}{l}
-y_{1}^{\prime \prime}=\lambda y_{1} \text { in }(0, \pi), \\
q y_{1}-\nu y_{2}^{\prime \prime}=\lambda y_{2} \text { in }(0, \pi), \\
y_{1}(0)=y_{2}(0)=0, \quad y_{1}(\pi)=y_{2}(\pi)=0
\end{array}\right.
$$


If $y_{1} \equiv 0$, then, $\lambda=\nu k^{2}$ is an eigenvalue of $L^{*}$ and taking $y_{2}=\varphi_{k}$, we obtain $\Phi_{2, k}^{*}$ as associated eigenfunction of $L^{*}$. Now assume that $y_{1} \not \equiv 0$, then $\lambda=k^{2}$ and $y_{1}=\varphi_{k}$ is a (normalized) solution to the first o.d.e. Inserting this expression in the second equation, we get for $y_{2}$ :

$$
\left\{\begin{array}{l}
y_{2}^{\prime \prime}+\frac{k^{2}}{\nu} y_{2}=\frac{1}{\nu} q \varphi_{k} \text { in }(0, \pi), \\
y_{2}(0)=y_{2}(\pi)=0
\end{array}\right.
$$

This proves that $\Phi_{1, k}^{*}$, is the second eigenfunction of $L^{*}$, associated to $k^{2}$. Moreover (2.1) (resp. (2.2)) can be deduce from (2.4) (resp. (2.6)).

Lemma 2.3. The sequence $\mathcal{B}^{*}=\left\{\Phi_{1, k}^{*}, \Phi_{2, k}^{*}: k \in \mathbb{N}^{*}\right\}$ is complete in $L^{2}\left(0, \pi ; \mathbb{R}^{2}\right)$.

Proof of Lemma 2.3.

Indeed, if $f=\left(f_{1}, f_{2}\right)$ is such that

$$
\left\langle f, \Phi_{\mu, k}^{*}\right\rangle=0, \quad \forall k \geq 1, \quad \forall \mu=1,2,
$$

then in particular

$$
\forall k \geq 1 \quad\left\{\begin{array}{l}
\left\langle f_{2}, \varphi_{k}\right\rangle=0 \\
\left\langle f_{1}, \varphi_{k}\right\rangle+\left\langle f_{2}, \psi_{k}\right\rangle=0
\end{array}\right.
$$

This implies that $f_{1}=f_{2}=0$ (since $\left\{\varphi_{k}\right\}_{k \geq 1}$ is an orthonormal basis in $L^{2}(0, \pi)$ and proves the completeness of $\mathcal{B}^{*}$.

Proposition 2.4. There exists $\sqrt{\nu} \notin \mathbb{Q}_{+}^{*}$ and $q \in L^{\infty}(0, \pi)$ such that $\mathcal{B}^{*}=\left\{\Phi_{1, k}^{*}, \Phi_{2, k}^{*}: k \in \mathbb{N}^{*}\right\}$ is not a Riesz basis for $L^{2}\left(0, \pi ; \mathbb{R}^{2}\right)$.

To prove this result we will need the following two lemmas.

Lemma 2.5. For a sequence $\left\{f_{k}\right\}_{k \geq 1}$ in Hilbert space $(\mathcal{H},\langle\rangle$,$) the following conditions are equivalent:$

1. $\left\{f_{k}\right\}_{k \geq 1}$ is a Riesz basis for $\mathcal{H}$.

2. $\left\{f_{k}\right\}_{k \geq 1}$ is complete, and its Gram matrix $\left(\left\langle f_{k}, f_{j}\right\rangle\right)_{k, j \geq 1}$ defines a bounded, invertible operator on $l^{2}(\mathbb{N})$ the space of square summable scalar sequences.

Proof of Lemma 2.5. See for instance [7], Theorem 3.6.6, page 66.

Lemma 2.6. For any $\sigma \in(0, \infty)$, there exist an irrational number $\nu>0$ and a sequence of rational numbers $\left\{k_{p}, j_{p}\right\}_{p \geq 0}$ such that $k_{p}$ and $j_{p}$ are co-prime positive integers, the sequences $\left\{k_{p}\right\}_{p \geq 0}$ and $\left\{j_{p}\right\}_{p \geq 0}$ are strictly increasing and

$$
\lim e^{k_{p}^{2+\sigma}}\left|\sqrt{\nu}-\frac{k_{p}}{j_{p}}\right|=0
$$

In particular, we deduce the existence of a positive constant $C>0$ such that

$$
\left|j_{p} \sqrt{\nu}-k_{p}\right| \leq C k_{p} e^{-k_{p}^{2+\sigma}}, \quad \forall p \geq 1 .
$$

Proof of Lemma 2.6 . See [2], Lemma 6.22, page 47.

We now have all the ingredients to prove Proposition 2.4. 
Proof of Proposition 2.4. The determinant of the Gram matrix associated to the normalized vectors of $\mathcal{B}^{*}$ is equal to

$$
\begin{aligned}
\operatorname{det}\left[G_{k_{p}, j_{p}}(\nu, q)\right] & =1-\frac{\left|\left\langle\Phi_{1, k}, \Phi_{2, j}\right\rangle\right|^{2}}{\left\|\Phi_{1, k}\right\|_{H_{0}^{1}}^{2}\left\|\Phi_{2, j}\right\|_{H_{0}^{1}}^{2}} \\
& =1-\frac{\left|\int_{0}^{\pi} \psi_{k}^{\prime}(x) \varphi_{j}^{\prime}(x) \mathrm{d} x\right|^{2}}{j^{2}\left(k^{2}+\left\|\psi_{k}\right\|_{H_{0}^{1}}^{2}\right)} \\
& =1-\frac{j^{2}\left|\int_{0}^{\pi} q(x) \varphi_{k}(x) \varphi_{j}(x) \mathrm{d} x\right|^{2}}{\left(k^{2}+\sum_{n \geq 1} \frac{n^{2}\left|\int_{0}^{\pi} q(x) \varphi_{k}(x) \varphi_{n}(x) \mathrm{d} x\right|^{2}}{\left|k^{2}-\nu n^{2}\right|^{2}}\left|k^{2}-\nu j^{2}\right|^{2}\right.} \\
& =1-\frac{1}{\left\{1+U_{k, j}(\nu, q)\left[k^{2}+V_{k, j}(\nu, q)\right]\right\}},
\end{aligned}
$$

where

$$
U_{k, j}(\nu, q)=\frac{\left|k^{2}-\nu j^{2}\right|^{2}}{j^{2}\left|\int_{0}^{\pi} q(x) \varphi_{k}(x) \varphi_{j}(x) \mathrm{d} x\right|^{2}}, \quad V_{k, j}(\nu, q)=\sum_{n \neq j} \frac{n^{2}\left|\int_{0}^{\pi} q(x) \varphi_{k}(x) \varphi_{n}(x) \mathrm{d} x\right|^{2}}{\left|k^{2}-\nu n^{2}\right|^{2}} .
$$

Remark that, there exists a constant $C\left(\nu,\|q\|_{L^{\infty}}\right)>0$ such that,

$$
V_{k, j}(\nu, q) \leq C\left(\nu,\|q\|_{L^{\infty}}\right), \quad \forall k, j \geq 1
$$

Thanks to Lemma 2.6, we can extract a subsequence $\left(k_{p}+j_{p}\right)_{p \geq 0}$ of even numbers only or odd numbers only, such that this two situations:

1. By choosing the subsequence of even numbers with $q(x)=\sin (x), x \in(0, \pi)$, we obtain

$$
\left|\int_{0}^{\pi} q(x) \varphi_{k_{p}}(x) \varphi_{j_{p}}(x) \mathrm{d} x\right|=\frac{2}{\pi} \frac{4 k_{p} j_{p}}{\left[\left(j_{p}-k_{p}\right)^{2}-1\right]\left[\left(j_{p}+k_{p}\right)^{2}-1\right]}
$$

Consequently

$$
k_{p}^{2} U_{k_{p}, j_{p}} \underset{p \longrightarrow+\infty}{\longrightarrow} 0, \text { thus } \operatorname{det}\left[G_{k_{p}, j_{p}}\right]_{p \longrightarrow+\infty}^{\longrightarrow} 0
$$

2. By choosing the subsequence of odd numbers with $q(x)=\sin (2 x), x \in(0, \pi)$, we obtain

$$
\left|\int_{0}^{\pi} q(x) \varphi_{k_{p}}(x) \varphi_{j_{p}}(x) \mathrm{d} x\right|=\frac{2}{\pi} \frac{8 k_{p} j_{p}}{\left|\left(j_{p}-k_{p}\right)^{2}-2\right|\left[\left(j_{p}+k_{p}\right)^{2}-2\right]} .
$$


Consequently

$$
k_{p}^{2} U_{k_{p}, j_{p}} \underset{p \longrightarrow+\infty}{\longrightarrow} 0, \text { thus } \operatorname{det}\left[G_{k_{p}, j_{p}}\right]_{p \longrightarrow+\infty}^{\longrightarrow} 0
$$

Indeed, take the first point (1) and let us fix $\sigma>0$ and $\sqrt{\nu}>0$. We have

$$
\begin{aligned}
k_{p} \sqrt{U_{k_{p}, j_{p}}} & =\frac{k_{p}\left|k_{p}^{2}-\nu j_{p}^{2}\right|}{j_{p}\left|\int_{0}^{\pi} q(x) \varphi_{k_{p}}(x) \varphi_{j_{p}}(x) \mathrm{d} x\right|} \\
& =\frac{\pi}{2} \frac{k_{p}\left|k_{p}+\sqrt{\nu} j_{p}\right|\left[\left(j_{p}-k_{p}\right)^{2}-1\right]\left[\left(j_{p}+k_{p}\right)^{2}-1\right]}{2 j_{p}^{2} k_{p}}\left|k_{p}-\sqrt{\nu} j_{p}\right| \\
& \leq \frac{\pi}{2} \frac{\left|k_{p}+\sqrt{\nu} j_{p}\right|\left[\left(j_{p}-k_{p}\right)^{2}-1\right]\left[\left(j_{p}+k_{p}\right)^{2}-1\right]}{2 j_{p}^{2} k_{p}} k_{p}^{2} e^{-k_{p}^{2+\sigma}} \underset{p \rightarrow+\infty}{\longrightarrow} 0 .
\end{aligned}
$$

Thanks to formula $(2.10)$,

$$
\operatorname{det}\left[G_{k_{p}, j_{p}}\right] \underset{p \longrightarrow+\infty}{\longrightarrow} 0
$$

The point (2) can be similarly shown.

Concerning the approximate controllability of system (1.1), it is well known that can be characterized in terms of a property of the solutions to (1.17). More precisely, system (1.1) is approximately controllable if and only if the following unique continuation property holds:

"Let $\theta_{0} \in H_{0}^{1}\left(0, \pi ; \mathbb{R}^{2}\right)$ be given and let $\theta$ be the associated adjoint state. Then, if $B^{*} D \theta_{x}(0, t)=0$ on $(0, T)$, one has $\theta_{0} \equiv 0$ in $Q_{T}$ ".

Fattorini gave an interesting characterization of the approximate controllability under a general abstract framework. In his paper [10], he proved that, under some reasonable assumptions, the only observation of the eigenfunctions completely characterizes the approximate controllability. Actually, this theorem has been proved for bounded observation operators but G. Olive (in [17]), give a generalization to the case of relatively bounded observation operators. We deduce that system (1.1) is approximately controllable at time $T>0$, if and only if for any $s \in \mathbb{C}$ and any $u \in \mathcal{D}\left(L^{*}\right)=H^{2}\left(0, \pi ; \mathbb{R}^{2}\right) \cap H_{0}^{1}\left(0, \pi ; \mathbb{R}^{2}\right)$ we have

$$
\left.\begin{array}{l}
L^{*} u=s u, \\
B^{*} D \partial_{x} u(0)=0
\end{array}\right\} \Longrightarrow u \equiv 0 \quad \text { in }(0, \pi) .
$$

This previous relation (2.11) justifies the second point of the Theorem 1.1 and will be used to prove the approximate controllability of system (1.1).

\subsection{Proof of Theorem 1.1: first point (a)}

In this subsection, our objective is to prove that system (1.1) is null controllable at time $T$ if $T>\widetilde{T}_{0} \in[0, \infty)$, when $\sqrt{\nu}>1$. If $y$ is the solution of system (1.1) associated with $y_{0} \in H^{-1}\left(0, \pi ; \mathbb{R}^{2}\right)$ and $u \in L^{2}(0, T)$, then it can be checked that $y(T)=0$ in $(0, \pi)$ if and only if

$$
\int_{0}^{T} u(t) B^{*} D \theta_{x}(0, t) \mathrm{d} t=-\left\langle y_{0}, \theta(\cdot, 0)\right\rangle_{H^{-1}, H_{0}^{1}}, \quad \forall \theta_{0} \in H_{0}^{1}\left(0, \pi ; \mathbb{R}^{2}\right)
$$


where $\theta$ is the solution of the adjoint problem (1.17) associated with $\theta_{0}$. Taking

$$
\theta_{0}:=\Phi_{i, k}^{*}, \forall k \geq 1, i=1,2
$$

the corresponding solution to the adjoint problem (1.17) is given by

$$
\left\{\begin{array}{l}
\theta^{1, k}(x, t)=e^{-k^{2}(T-t)} \Phi_{1, k}^{*}(x), \\
\theta^{2, k}(x, t)=e^{-\nu k^{2}(T-t)} \Phi_{2, k}^{*}(x), \forall k \geq 1 .
\end{array}\right.
$$

Since the sequence $\mathcal{B}^{*}=\left\{\Phi_{1, k}^{*}, \Phi_{2, k}^{*}: k \in \mathbb{N}^{*}\right\}$ is complete in $L^{2}\left(0, \pi ; \mathbb{R}^{2}\right)$, the null controllability problem for system (1.1) is equivalent to find $u \in L^{2}(0, T)$ such that:

$$
\left\{\begin{array}{l}
\int_{0}^{T} e^{-k^{2} t} u(T-t) \mathrm{d} t=-e^{-k^{2} T}\left\langle y_{0}, \psi_{1, k}\right\rangle_{H^{-1}, H_{0}^{1}}, \\
\int_{0}^{T} e^{-\nu k^{2} t} u(T-t) \mathrm{d} t=-e^{-\nu k^{2} T}\left\langle y_{0}, \psi_{2, k}\right\rangle_{H^{-1}, H_{0}^{1}}, \quad \forall k \geq 1,
\end{array}\right.
$$

where $\psi_{1, k}$ and $\psi_{2, k}$ are defined in (1.9). We are now going to give some results that will be crucial, to solve (2.13). One has:

Proposition 2.7. Let $\sqrt{\nu}>1$. Let us define

$$
\begin{aligned}
I: \mathbb{N}^{*} & \longrightarrow \mathbb{N}^{*} \\
k & \longmapsto i_{k}
\end{aligned}
$$

where, for any $k \in \mathbb{N}^{*}, i_{k}$ is the nearest integer to $\sqrt{\nu} k$ i.e

$$
\left|\sqrt{\nu} k-i_{k}\right|<\frac{1}{2}
$$

Thus for any $k \in \mathbb{N}^{*}$,

$$
|\sqrt{\nu} k-i|>\frac{1}{2}, \quad \forall i \in \mathbb{N}^{*}, i \neq i_{k}
$$

Then

1. The function $I$ is injective.

2. $\widehat{I}=\mathbb{N}^{*} \backslash I\left(\mathbb{N}^{*}\right)=\left\{\hat{i}_{k}: k \geq 1\right\}$, is a infinite set, where the elements of $\widehat{I}$ are classified in ascending order.

Proof of Proposition 2.\%.

1. Let us assume that $I\left(k_{1}\right)=I\left(k_{2}\right)$, where $k_{1}, k_{2} \in \mathbb{N}^{*}$. We have $i_{k_{1}}=i_{k_{2}}$ with $\left|\sqrt{\nu} k_{1}-i_{k_{1}}\right|<\frac{1}{2}$ and $\left|\sqrt{\nu} k_{2}-i_{k_{2}}\right|<\frac{1}{2}$. This leads to

$$
-1+\left(i_{k_{1}}-i_{k_{2}}\right)<\sqrt{\nu}\left(k_{1}-k_{2}\right)<1+\left(i_{k_{1}}-i_{k_{2}}\right)
$$

and thus $\left|k_{1}-k_{2}\right|<\frac{1}{\sqrt{\nu}}<1$, which implies $k_{1}=k_{2}$. 
2. Assume $\sqrt{\nu}>1, \sqrt{\nu} \notin \mathbb{Q}_{+}^{*}$.

- If $\sqrt{\nu}>2$ then, $\forall n \in \mathbb{N}^{*}, i_{n+1}-i_{n}>1$.

- Suppose $1<\sqrt{\nu}<2$. There exists a sequence of integers $\left(n_{k}\right)_{k \in \mathbb{N}^{*}}$ strictly increasing, such that $i_{n_{k}+1}-$ $i_{n_{k}}>1$. Actually, let us take for instance

$$
\left.n_{k} \in\right] \frac{2 k+1}{2(\sqrt{\nu}-1)}-1, \frac{2 k+1}{2(\sqrt{\nu}-1)}[, \quad k \geq 1
$$

We deduce

$$
-\frac{1}{2}<\frac{1}{2}-\sqrt{\nu}+1<n_{k} \sqrt{\nu}-\left(n_{k}+k\right)<\frac{1}{2}, \quad \text { i.e } \quad i_{n_{k}}=n_{k}+k .
$$

Moreover

$$
\begin{aligned}
i_{n_{k}+1}-i_{n_{k}}=i_{n_{k}+1}-n_{k}-k & >\sqrt{\nu}\left(n_{k}+1\right)-\frac{1}{2}-n_{k}-k \\
& =n_{k} \sqrt{\nu}-\left(n_{k}+k\right)+\sqrt{\nu}-\frac{1}{2} \\
& >\frac{1}{2}-\sqrt{\nu}+1+\sqrt{\nu}-\frac{1}{2}=1 .
\end{aligned}
$$

\subsubsection{Positive null controllability result}

Thanks to Proposition 2.7 we can reformulate (2.13). We say that the null controllability property at time $T$ for system (1.1) is equivalent to find $u \in L^{2}(0, T)$ such that:

$$
\left\{\begin{array}{l}
\int_{0}^{T} e^{-\widehat{i}_{k}^{2} t} u(T-t) \mathrm{d} t=-e^{-\widehat{i}_{k}^{2} T}\left\langle y_{0}, \psi_{1, \widehat{i}_{k}}\right\rangle_{-1,1}, \\
\int_{0}^{T} e^{-i_{k}^{2} t} u(T-t) \mathrm{d} t=-e^{-i_{k}^{2} T}\left\langle y_{0}, \psi_{1, i_{k}}\right\rangle_{-1,1}, \quad \forall k \geq 1 . \\
\int_{0}^{T} e^{-\nu k^{2} t} u(T-t) \mathrm{d} t=-e^{-\nu k^{2} T}\left\langle y_{0}, \psi_{2, k}\right\rangle_{-1,1},
\end{array}\right.
$$

We can now state our following main result.

Proposition 2.8. Let us introduce the (closed) space $E_{T} \subset L^{2}(0, T)$ given by

$$
E_{T}=\overline{\operatorname{span}\left\{e^{-k^{2} t}, e^{-\nu k^{2} t}: k \geq 1\right\}} L^{2}(0, T) .
$$

Then 
1. There exists a family $\left\{q_{k}\right\}_{k \geq 1} \subset E_{T}$ such that

$$
\left\{\begin{array}{l}
\int_{0}^{T} e^{-\widehat{i}_{k}^{2} t} q_{k}(t) \mathrm{d} t=-e^{-\widehat{i}_{k}^{2} T}\left\langle y_{0}, \psi_{1, \hat{i}_{k}}\right\rangle_{-1,1}, \\
\int_{0}^{T} e^{-i_{k}^{2} t} q_{k}(t) \mathrm{d} t=-e^{-i_{k}^{2} T}\left\langle y_{0}, \psi_{1, i_{k}}\right\rangle_{-1,1}, \\
\int_{0}^{T} e^{-\nu k^{2} t} q_{k}(t) \mathrm{d} t=-e^{-\nu k^{2} T}\left\langle y_{0}, \psi_{2, k}\right\rangle_{-1,1}, \quad k \geq 1, \\
\int_{0}^{T} e^{-\widehat{i}_{k}^{2} t} q_{j}(t) \mathrm{d} t=\int_{0}^{T} e^{-i_{k}^{2} t} q_{j}(t) \mathrm{d} t=\int_{0}^{T} e^{-\nu k^{2} t} q_{j}(t) \mathrm{d} t=0, \quad k \neq j .
\end{array}\right.
$$

2. If $T>\widetilde{T}_{0}=\max \left\{T_{1}, \widetilde{T}_{2}\right\}$ (see (1.12)) then we infer that an explicit solution u of moment problem (2.14) is given by

$$
u(t)=\sum_{k \geq 1} q_{k}(T-t)
$$

Proof of Proposition 2.8. Let us start by recalling classical properties of the Laplace transform (see for instance [19], pp.19-20). Let $H^{2}\left(\mathbb{C}_{+}\right)$the space of holomorphic functions $\Phi$ on $\mathbb{C}_{+}=\{z \in \mathbb{C}, \Re(z)>0\}$ such that

$$
\sup _{\sigma>0}\|\Phi(\sigma+i \bullet)\|_{L^{2}(\mathbb{R} ; \mathbb{C})}<\infty
$$

endowed with the norm

$$
\|\Phi\|_{H^{2}\left(\mathbb{C}_{+}\right)}^{2}=\sup _{\sigma>0}\|\Phi(\sigma+i \bullet)\|_{L^{2}(\mathbb{R} ; \mathbb{C})}^{2}=\int_{\mathbb{R}}|\Phi(i \tau)|^{2} \mathrm{~d} \tau
$$

Then the Laplace transform

$$
\begin{aligned}
L: L^{2}(0,+\infty ; \mathbb{C}) & \longrightarrow \\
f & \longmapsto\left(\Phi \lambda \in \mathbb{C}^{+} \longmapsto \int_{\mathbb{R}} e^{-\lambda t} f(t) \mathrm{d} t\right)
\end{aligned}
$$

is an isomorphism. In the sequel, We shall construct for each $k$, a function $J_{k} \in H^{2}\left(\mathbb{C}_{+}\right)$satisfying appropriate properties, and take advantage of the isomorphism property of the Laplace transform to conclude. Let us fix $k \geq 1$, we define $J_{k}$, for $\lambda \in \mathbb{C}_{+}$, as

$$
J_{k}(\lambda)=\left\{\alpha_{k}\left(\lambda-i_{k}^{2}\right)\left(\lambda-\widehat{i}_{k}^{2}\right)+\beta_{k}\left(\lambda-\nu k^{2}\right)\left(\lambda-\widehat{i}_{k}^{2}\right)+\gamma_{k}\left(\lambda-\nu k^{2}\right)\left(\lambda-i_{k}^{2}\right)\right\} L_{k}(\lambda)
$$

where $\alpha_{k}, \beta_{k}$ and $\gamma_{k}$ are constants to be determined and $L_{k}$ is the following Blaschke-type product

$$
L_{k}(\lambda)=\frac{1}{(1+\lambda)^{3}} \prod_{j \geq 1, j \neq k}\left(\frac{\lambda-\nu j^{2}}{\lambda+\nu j^{2}}\right)\left(\frac{\lambda-i_{j}^{2}}{\lambda+i_{j}^{2}}\right)\left(\frac{\lambda-\widehat{i}_{j}^{2}}{\lambda+\widehat{i}_{j}^{2}}\right) \text {. }
$$


Notice that for any $k \geq 1$,

$$
\left|L_{k}(i \tau)\right|^{2}=\frac{1}{\left(1+\tau^{2}\right)^{3}}, \forall \tau \in \mathbb{R}
$$

This implies $J_{k} \in H^{2}\left(\mathbb{C}_{+}\right)$, moreover

$$
J_{k}\left(\nu j^{2}\right)=J_{k}\left(i_{j}^{2}\right)=J_{k}\left(\widehat{i}_{j}^{2}\right)=0, \quad \forall j \geq 1, j \neq k .
$$

So, using that the Laplace transform is a isomorphism from $L^{2}(0, \infty ; \mathbb{C})$ into $H^{2}\left(\mathbb{C}_{+}\right)$, we infer the existence of a nontrivial function $\tilde{q}_{k} \in L^{2}(0, \infty ; \mathbb{C})$ such that

$$
J_{k}(\lambda)=\int_{0}^{+\infty} e^{-\lambda t} \widetilde{q}_{k}(t) \mathrm{d} t, \quad \forall \lambda \in \mathbb{C}_{+} .
$$

Now, we can choose $\alpha_{k}, \beta_{k}$ and $\gamma_{k}$ such that

$$
\left\{\begin{array}{l}
J_{k}\left(\nu k^{2}\right)=-e^{-\nu k^{2} T}\left\langle y^{0}, \psi_{2, k}\right\rangle_{-1,1}, \\
J_{k}\left(i_{k}^{2}\right)=-e^{-i_{k}^{2} T}\left\langle y^{0}, \psi_{1, i_{k}}\right\rangle_{-1,1}, \quad \forall k \geq 1 . \\
J_{k}\left(\widehat{i}_{k}^{2}\right)=-e^{-\widehat{i}_{k}^{2} T}\left\langle y^{0}, \psi_{1, \widehat{i}_{k}}\right\rangle_{-1,1},
\end{array}\right.
$$

We obtain

$$
\left\{\begin{array}{l}
\alpha_{k}=-\frac{e^{-\nu k^{2} T}}{\left(\nu k^{2}-i_{k}^{2}\right)\left(\nu k^{2}-\widehat{i}_{k}^{2}\right) L_{k}\left(\nu k^{2}\right)}\left\langle y_{0}, \psi_{2, k}\right\rangle_{-1,1}, \\
\beta_{k}=-\frac{e^{-i_{k}^{2} T}}{\left(i_{k}^{2}-\nu k^{2}\right)\left(i_{k}^{2}-\widehat{i}_{k}^{2}\right) L_{k}\left(i_{k}^{2}\right)}\left\langle y_{0}, \psi_{1, i_{k}}\right\rangle_{-1,1}, \quad \forall k \geq 1 . \\
\gamma_{k}=-\frac{e^{-\widehat{i}_{k}^{2} T}}{\left(\hat{i}_{k}^{2}-\nu k^{2}\right)\left(\hat{i}_{k}^{2}-i_{k}^{2}\right) L_{k}\left(\hat{i}_{k}^{2}\right)}\left\langle y_{0}, \psi_{1, \widehat{i}_{k}}\right\rangle_{-1,1},
\end{array}\right.
$$

The Parseval equality gives

$$
\begin{aligned}
\left\|\widetilde{q}_{k}\right\|_{L^{2}(0, \infty ; \mathbb{C})}^{2} & =\int_{-\infty}^{+\infty}\left|J_{k}(i \tau)\right|^{2} \mathrm{~d} \tau \\
& =\int_{-\infty}^{+\infty} \frac{\left|\left(i \tau-\widehat{i}_{k}^{2}\right)\left\{\left(\alpha_{k}+\beta_{k}\right)\left(i \tau-i_{k}^{2}\right)+\beta_{k}\left(i_{k}^{2}-\nu k^{2}\right)\right\}+\gamma_{k}\left(i \tau-\nu k^{2}\right)\left(i \tau-i_{k}^{2}\right)\right|^{2}}{|1+i \tau|^{6}} \mathrm{~d} \tau, \\
& =\int_{-\infty}^{+\infty} \frac{\left|\left(\alpha_{k}+\beta_{k}\right)\left(i \tau-i_{k}^{2}\right)\left(i \tau-\widehat{i}_{k}^{2}\right)+\beta_{k}\left(i_{k}^{2}-\nu k^{2}\right)\left(i \tau-\widehat{i}_{k}^{2}\right)+\gamma_{k}\left(i \tau-\nu k^{2}\right)\left(i \tau-i_{k}^{2}\right)\right|^{2}}{\left|1+\tau^{2}\right|^{3}} \mathrm{~d} \tau, \\
& \leq C \nu^{2} k^{4} i_{k}^{4} \widehat{i}_{k}^{4}\left\{\left(\alpha_{k}+\beta_{k}\right)^{2}+\beta_{k}^{2}\left(i_{k}^{2}-\nu k^{2}\right)^{2}+\gamma_{k}^{2}\right\} .
\end{aligned}
$$

In the sequel, we will show that there exists $q_{k} \in L^{2}(0, T)$ such that

$$
\left\|q_{k}\right\|_{L^{2}(0, T)}^{2} \leq C \nu^{2} k^{4} i_{k}^{4} \widehat{i}_{k}^{4}\left\{\left(\alpha_{k}+\beta_{k}\right)^{2}+\beta_{k}^{2}\left(i_{k}^{2}-\nu k^{2}\right)^{2}+\gamma_{k}^{2}\right\} .
$$


Assume that a countable family $\Lambda$ satisfies the weak gap condition. ${ }^{1}$ On one hand, let us consider the closed space $A(\Lambda, T) \subset L^{2}(0, T ; \mathbb{C})$ given by

$$
A(\Lambda, T)={\overline{\operatorname{Span}\left\{e^{-\lambda t} ; \lambda \in \Lambda\right\}}}^{L^{2}(0, T ; \mathbb{C})} .
$$

For any $T \in(0,+\infty)$, the restriction operator

$$
R_{\Lambda, T}: \phi \in A(\Lambda, \infty) \rightarrow R_{\Lambda, T} \phi=\phi_{\mid(0, T)} \in A(\Lambda, T)
$$

is an isomorphism. Moreover there exists a constant $C_{T}$ such that

$$
\left\|R_{\Lambda, T}^{-1}\right\| \leq C_{T} .
$$

Indeed, the fact that $R_{\Lambda, T}$ is an isomorphism is proved in [18]. The proof of the bound (2.20) can be done by contradiction (see [2]. Lem. 4.2 and [5], Prop. 2.4).

On the other hand, for any $T>0$, there exists a constant $C_{T}$ such that for any $\widetilde{q} \in L^{2}(0,+\infty ; \mathbb{C})$ there exists $q \in L^{2}(0, T ; \mathbb{C})$ satisfying

$$
\int_{0}^{T} q(t) e^{-\lambda t} \mathrm{~d} t=\int_{0}^{+\infty} \widetilde{q}(t) e^{-\lambda t} \mathrm{~d} t, \quad \forall \lambda \in \Lambda,
$$

and

$$
\|q\|_{L^{2}(0, T, \mathbb{C})} \leq C_{T}\|\widetilde{q}\|_{L^{2}(0,+\infty, \mathbb{C})} .
$$

Indeed, from [1] Corollary 4.3, it comes that $A(\Lambda, \infty)$ is a proper subspace of $L^{2}(0,+\infty ; \mathbb{C})$. Let $\Pi_{\Lambda}$ the associated orthogonal projection and $\widetilde{q} \in L^{2}(0,+\infty ; \mathbb{C})$. Then, by construction, we have

$$
\int_{0}^{+\infty} \Pi_{\Lambda} \widetilde{q}(t) e^{-\lambda t} \mathrm{~d} t=\int_{0}^{+\infty} \widetilde{q}(t) e^{-\lambda t} \mathrm{~d} t, \quad \forall \lambda \in \Lambda .
$$

Since the restriction operator $R_{\Lambda, T}$ defined in (2.19) is an isomorphism, choose $q:=\left(R_{\Lambda, T}^{-1}\right)^{*} \Pi_{\Lambda} \widetilde{q}$, thus, there exists $C_{T}$ such that

$$
\|q\|_{L^{2}(0, T, \mathbb{C})} \leq C_{T}\|\widetilde{q}\|_{L^{2}(0,+\infty, \mathbb{C})}
$$

Using (2.21), for every $\lambda \in \Lambda$

$$
\begin{aligned}
\int_{0}^{T} q(t) e^{-\lambda t} \mathrm{~d} t & =\left\langle\left(R_{\Lambda, T}^{-1}\right)^{*} \Pi_{\Lambda} \widetilde{q}(\cdot), e^{-\lambda \cdot}\right\rangle_{L^{2}(0, T)}, \\
& =\left\langle\Pi_{\Lambda} \widetilde{q}(\cdot), R_{\Lambda, T}^{-1} R_{\Lambda, T} e^{-\lambda \cdot}\right\rangle_{L^{2}(0,+\infty)}, \\
& =\int_{0}^{T} \widetilde{q}_{k}(t) e^{-\lambda t} \mathrm{~d} t .
\end{aligned}
$$

This proves inequality (2.18).

\footnotetext{
${ }^{1}$ This notion was introduced in [5].
} 
Now, thanks to equalities (2.17), we obtain for all $k \geq 1$ :

$$
\left\{\begin{array}{l}
\left(\alpha_{k}+\beta_{k}\right)^{2}=\frac{1}{\left(\nu k^{2}-i_{k}^{2}\right)^{2}}\left|\left\langle y^{0}, \frac{e^{-\nu k^{2} T} \psi_{2, k}}{\left(\nu k^{2}-\widehat{i}_{k}^{2}\right) L_{k}\left(\nu k^{2}\right)}-\frac{e^{-i_{k}^{2} T} \psi_{1, i_{k}}}{\left(i_{k}^{2}-\widehat{i}_{k}^{2}\right) L_{k}\left(i_{k}^{2}\right)},\right\rangle_{-1,1}\right|^{2}, \\
\beta_{k}^{2}\left(i_{k}^{2}-\nu k^{2}\right)^{2}+\gamma_{k}^{2}=\frac{1}{\left.\widehat{i}_{k}^{2}-i_{k}^{2}\right)^{2}}\left(\frac{e^{-2 i_{k}^{2} T}\left|\left\langle y^{0}, \psi_{1, i_{k}}\right\rangle_{-1,1}\right|^{2}}{\left|L_{k}\left(i_{k}^{2}\right)\right|^{2}}+\frac{e^{-2 \widehat{i}_{k}^{2} T}\left|\left\langle y^{0}, \psi_{1, \widehat{i}_{k}}\right\rangle_{-1,1}\right|^{2}}{\left(\widehat{i}_{k}^{2}-\nu k^{2}\right)^{2}\left|L_{k}\left(\widehat{i}_{k}^{2}\right)\right|^{2}}\right)^{2} .
\end{array}\right.
$$

Thus the control $u$ defines an element of $L^{2}(0, T)$ if

$$
U=\sum_{k \geq 1} U_{k}=\sum_{k \geq 1} \frac{\left(k i_{k} \widehat{i}_{k}\right)^{4}}{\left(\nu k^{2}-i_{k}^{2}\right)^{2}}\left|\left\langle y^{0}, \frac{e^{-\nu k^{2} T} \psi_{2, k}}{\left(\nu k^{2}-\widehat{i}_{k}^{2}\right) L_{k}\left(\nu k^{2}\right)}-\frac{e^{-i_{k}^{2} T} \psi_{1, i_{k}}}{\left(i_{k}^{2}-\widehat{i}_{k}^{2}\right) L_{k}\left(i_{k}^{2}\right)}\right\rangle_{-1,1}\right|^{2}<+\infty
$$

and

$$
V=\sum_{k \geq 1} V_{k}=\sum_{k \geq 1} \frac{\left(k i_{k} \widehat{i}_{k}\right)^{4}}{\left(\hat{i}_{k}^{2}-i_{k}^{2}\right)^{2}}\left(\frac{e^{-2 i_{k}^{2} T}\left|\left\langle y^{0}, \psi_{1, i_{k}}\right\rangle_{-1,1}\right|^{2}}{\left|L_{k}\left(i_{k}^{2}\right)\right|^{2}}+\frac{e^{-2 \widehat{i}_{k}^{2} T}\left|\left\langle y^{0}, \psi_{1, \widehat{i}_{k}}\right\rangle_{-1,1}\right|^{2}}{\left.\left(\hat{i}_{k}^{2}-\nu k^{2}\right)^{2} \mid L_{k} \widehat{(i}_{k}^{2}\right)\left.\right|^{2}}\right)^{2}<+\infty
$$

Let us give first some properties of $L_{k}$ (see (2.16)).

Lemma 2.9. Assume $\sqrt{\nu}>1, \sqrt{\nu} \notin \mathbb{Q}_{+}^{*}$.

1. For every $\varepsilon>0$ there exists positive constants $C_{1}(\varepsilon), C_{2}(\varepsilon)$, and $C_{3}(\varepsilon)$, independent of $k$, such that

$$
\left|L_{k}\left(i_{k}^{2}\right)\right| \geq C_{1}(\varepsilon) e^{-\varepsilon i_{k}^{2}},\left|L_{k}\left(\widehat{i}_{k}^{2}\right)\right| \geq C_{2}(\varepsilon) e^{-\varepsilon \hat{i}_{k}^{2}}, \text { and }\left|L_{k}\left(\nu k^{2}\right)\right| \geq C_{3}(\varepsilon) e^{-\varepsilon \nu k^{2}}, \forall k \geq 1 .
$$

2. There exists a constant $C>0$, independent of $k$, such that

$$
\left|L_{k}^{\prime}(\lambda)\right| \leq C, \quad \forall \lambda \geq 0
$$

Proof of Lemma 2.9.

1. Let us fix $k \geq 1$ and work with $L_{k}\left(\nu k^{2}\right)$. We have

$$
\left|L_{k}\left(\nu k^{2}\right)\right|=\frac{1}{\left(1+\nu k^{2}\right)^{3}} \prod_{\substack{j \geq 1 \\ j \neq k}} \frac{\left|1-\frac{\nu k^{2}}{\nu j^{2}}\right|}{\left(1+\frac{\nu k^{2}}{\nu j^{2}}\right)} \frac{\left|1-\frac{\nu k^{2}}{i_{j}^{2}}\right|}{\left(1+\frac{\nu k^{2}}{i_{j}^{2}}\right)} \frac{\left|1-\frac{\nu k^{2}}{\widehat{i}_{j}^{2}}\right|}{\left(1+\frac{\nu k^{2}}{\hat{i}_{j}^{2}}\right)} .
$$

- Let us fix $\varepsilon>0$, there exists $N(\varepsilon)$ such that

$$
\sum_{j \geq N(\varepsilon)}\left(\frac{1}{\nu j^{2}}+\frac{1}{i_{j}^{2}}+\frac{1}{\hat{i}_{j}^{2}}\right) \leq \varepsilon
$$


Using the inequality $1+x \leq e^{x}, x \in \mathbb{R}$, we can estimate the denominator of (2.24) as follows:

$$
\begin{aligned}
& \left(1+\nu k^{2}\right)^{3} \prod_{j \geq 1, j \neq k}\left(1+\frac{\nu k^{2}}{\nu j^{2}}\right)\left(1+\frac{\nu k^{2}}{i_{j}^{2}}\right)\left(1+\frac{\nu k^{2}}{\widehat{i}_{j}^{2}}\right) \\
& \leq\left(1+\nu k^{2}\right)^{3} \prod_{j=1}^{N(\varepsilon)-1}\left(1+\nu k^{2}\right)^{3} \prod_{j \geq N(\varepsilon)}\left(1+\frac{\nu k^{2}}{\nu j^{2}}\right)\left(1+\frac{\nu k^{2}}{i_{j}^{2}}\right)\left(1+\frac{\nu k^{2}}{\widehat{i}_{j}^{2}}\right) \\
& \leq\left(1+\nu k^{2}\right)^{3} \prod_{j=1}^{N(\varepsilon)-1}\left(1+\nu k^{2}\right)^{3} \exp \left\{\nu k^{2} \sum_{j \geq N(\varepsilon)}\left(\frac{1}{\nu j^{2}}+\frac{1}{i_{j}^{2}}+\frac{1}{\hat{i}_{j}^{2}}\right)\right\} \\
& \leq\left(1+\nu k^{2}\right)^{3 N(\varepsilon)} e^{\varepsilon \nu k^{2}} \leq C(\varepsilon) e^{\varepsilon \nu k^{2}}, \quad \forall k \in \mathbb{N}^{*},
\end{aligned}
$$

for a positive constant $C(\varepsilon)$.

- Let us now work on the numerator of (2.24).

Let us recall that (see Prop. 2.7), for all $k, j \geq 1$ with $j \neq k$, we have

$$
|\sqrt{\nu} j-\sqrt{\nu} k| \geq \sqrt{\nu}, \quad\left|i_{j}-\sqrt{\nu} k\right|>\frac{1}{2} \quad \text { and } \quad\left|\widehat{i}_{j}-\sqrt{\nu} k\right|>\frac{1}{2},
$$

we deduce that, the conditions (1.8) et (1.9) of Theorem 1.1 in [12] are satisfied. Thus for every $\varepsilon>0$ there exists positive constants $C_{1}(\varepsilon), C_{2}(\varepsilon)$ and $C_{3}(\varepsilon)$ such that

$$
\prod_{j \geq 1, j \neq k}\left|1-\frac{\nu k^{2}}{\nu j^{2}}\right| \geq C_{1}(\varepsilon) e^{-\varepsilon \nu k^{2}}, \prod_{j \geq 1, j \neq k}\left|1-\frac{\nu k^{2}}{i_{j}^{2}}\right| \geq C_{2}(\varepsilon) e^{-\varepsilon \nu k^{2}} \text { and } \prod_{j \geq 1, j \neq k}\left|1-\frac{\nu k^{2}}{i_{j}^{2}}\right| \geq C_{3}(\varepsilon) e^{-\varepsilon \nu k^{2}} .
$$

2. For all $\lambda \geq 0$, one has

$$
\frac{L_{k}^{\prime}(\lambda)}{L_{k}(\lambda)}=\frac{d}{d \lambda} \log L_{k}(\lambda)=\frac{-3}{1+\lambda}+\sum_{j \geq 1, j \neq k}\left(\frac{2 \widehat{i}_{j}^{2}}{\left(\lambda+\widehat{i}_{j}^{2}\right)^{2}} \frac{\lambda+\widehat{i}_{j}^{2}}{\lambda-\widehat{i}_{j}^{2}}+\frac{2 \nu j^{2}}{\left(\lambda+\nu j^{2}\right)^{2}} \frac{\lambda+\nu j^{2}}{\lambda-\nu j^{2}}+\frac{2 i_{j}^{2}}{\left(\lambda+i_{j}^{2}\right)^{2}} \frac{\lambda+i_{j}^{2}}{\lambda-i_{j}^{2}}\right)
$$

consequently

$$
\left|L_{k}^{\prime}(\lambda)\right| \leq 3+2 \sum_{j=1}^{+\infty}\left(\frac{1}{\hat{i}_{j}^{2}}+\frac{1}{\nu j^{2}}+\frac{1}{i_{j}^{2}}\right)<+\infty
$$


Let us now show that $V$ (see (2.23)) is a convergent series, when $T>T_{1}$ (see (1.12)). One has

$$
\begin{aligned}
V & \leq C \sum_{k \geq 1} k^{4} i_{k}^{4} \widehat{i}_{k}^{4}\left(\frac{e^{-2 i_{k}^{2} T}\left|\left\langle y_{0}, \psi_{1, i_{k}}\right\rangle_{-1,1}\right|^{2}}{\left|L_{k}\left(i_{k}^{2}\right)\right|^{2}}+\frac{e^{-2 \widehat{i}_{k}^{2} T}\left|\left\langle y_{0}, \psi_{1, \widehat{i}_{k}}\right\rangle_{-1,1}\right|^{2}}{\left.\mid L_{k} \widehat{i}_{k}^{2}\right)\left.\right|^{2}}\right) \\
& \leq C(\varepsilon)\left\|y_{0}\right\|_{-1}^{2} \sum_{k \geq 1} k^{4} i_{k}^{4} \widehat{i}_{k}^{4}\left(e^{-2 i_{k}^{2}(T-\varepsilon)}\left\|\psi_{1, i_{k}}\right\|^{2}+e^{-2 \hat{i}_{k}^{2}(T-\varepsilon)}\left\|\psi_{1, \hat{i}_{k}}\right\|^{2}\right) \\
& \leq C(\varepsilon)\left\|y_{0}\right\|_{-1}^{2} \sum_{k \geq 1} k^{4} i_{k}^{4} \widehat{i}_{k}^{4} e^{-2 k^{2}(T-\varepsilon)}\left\|\psi_{1, k}\right\|^{2} \\
& \left.=C(\varepsilon)\left\|y_{0}\right\|_{H-1}^{2} \sum_{k \geq 1} k^{4} i_{k}^{4} \widehat{i}_{k}^{4} e^{-2 k^{2}\left(T-\frac{\log \| \psi_{1, k}}{k^{2}}\right.}-\varepsilon\right) \\
& =C(\varepsilon)\left\|y_{0}\right\|_{H}^{2-1} \sum_{k \geq 1} k^{4} i_{k}^{4} \widehat{i}_{k}^{4} e^{-2 k^{2}\left(T-T_{1}-2 \varepsilon\right)}
\end{aligned}
$$

If we take, $\varepsilon \in\left(0, \frac{T-T_{1}}{2}\right)$, we deduce that $V$ converges if $T>T_{1}$. Let us see now that $U$ (see (2.22)) is a convergent series, when $T>\widetilde{T}_{2}$ (see (1.12)). One has, for all $k \geq 1 \mathrm{~s}$

$$
\begin{aligned}
& U_{k}=\frac{k^{4} i_{k}^{\hat{i}_{k}^{4}}}{\left(\nu k^{2}-i_{k}^{2}\right)^{2}}\left|\left\langle y_{0}, \frac{e^{-i_{k}^{2} T} \psi_{1, i_{k}}}{\left(i_{k}^{2}-\widehat{i}_{k}^{2}\right) L_{k}\left(i_{k}^{2}\right)}-\frac{e^{-\nu k^{2} T} \psi_{2, k}}{\left(\nu k^{2}-\widehat{i}_{k}^{2}\right) L_{k}\left(\nu k^{2}\right)}\right\rangle\right|^{2}, \\
& \leq \frac{k^{4} i_{k}^{4} \widehat{i}_{k}^{4}\left\|y_{0}\right\|_{-1}^{2}}{\left(\nu k^{2}-i_{k}^{2}\right)^{2}}\left\|\frac{e^{-i_{k}^{2} T}\left(\psi_{1, i_{k}}-\psi_{2, k}\right)}{\left(i_{k}^{2}-\widehat{i}_{k}^{2}\right) L_{k}\left(i_{k}^{2}\right)}+\left(\frac{e^{-i_{k}^{2} T}}{\left(i_{k}^{2}-\widehat{i}_{k}^{2}\right) L_{k}\left(i_{k}^{2}\right)}-\frac{e^{-\nu k^{2} T}}{\left(\nu k^{2}-\widehat{i}_{k}^{2}\right) L_{k}\left(\nu k^{2}\right)}\right) \psi_{2, k}\right\|^{2}, \\
& \leq k^{4} i_{k}^{4} \widehat{i}_{k}^{4} C(\varepsilon)\left\|y_{0}\right\|_{-1}^{2}\left(\exp \left\{-2 i_{k}^{2}\left[T-\frac{\log \frac{\left\|\psi_{2, k}-\psi_{1, i_{k}}\right\|}{\left|\nu k^{2}-i_{k}^{2}\right|}}{i_{k}^{2}}-\varepsilon\right]\right\}+e^{-2 \lambda_{k}^{*}\left(T-\frac{i_{k}^{2}}{\left.\lambda_{k}^{*} \varepsilon\right)}\right.}+e^{-2 \nu k^{2}\left(T-\varepsilon-\frac{i_{k}^{2}}{\left.\nu k^{2} \varepsilon\right)}\right)}\right), \\
& \leq k^{4} i_{k}^{4} \widehat{i}_{k}^{4} C\left(\varepsilon^{\prime}\right)\left\|y_{0}\right\|_{-1}^{2}\left(e^{-2 i_{k}^{2}\left[T-\widetilde{T}_{2}-\varepsilon^{\prime}\right]}+e^{-2 \lambda_{k}^{*}\left(T-2 \varepsilon^{\prime}\right)}+e^{-2 \nu k^{2}\left(T-\varepsilon^{\prime}\right)}\right),
\end{aligned}
$$

where $\lambda_{k}^{*}=\left(1-\theta_{k}\right) \nu k^{2}+\theta_{k} i_{k}^{2}$, with $\left.\theta_{k} \in\right] 0,1\left[\right.$. If we take, $\varepsilon^{\prime} \in\left(0, \frac{T-\widetilde{T}_{2}}{2}\right)$, we deduce that $U$ converge if $T>\widetilde{T}_{2}$. This gives the proof of null-controllability of system (1.1) if $T>\widetilde{T}_{0}=\max \left\{T_{1}, \widetilde{T}_{2}\right\}$.

\subsubsection{The negative null controllability result}

Let us prove that if $0<T<\widetilde{T}_{0}$, then system (1.1) is not null controllable at time $T$. We argue by contradiction. In particular, we assume that $\widetilde{T}_{0}>0$, otherwise there is nothing to prove. By Proposition 1.7, system (1.1) is null-controllable at time $T$ if and only if there exists $C>0$ such that any solution $\theta$ of the adjoint problem (1.17) satisfies the observability inequality:

$$
\|\theta(0)\|_{H_{0}^{1}\left(0, \pi ; \mathbb{R}^{2}\right)}^{2} \leq C \int_{0}^{T}\left|B^{*} D \theta_{x}(0, t)\right|^{2} \mathrm{~d} t, \quad \forall \theta^{0} \in H_{0}^{1}\left(0, \pi ; \mathbb{R}^{2}\right)
$$


Let us recall that

$$
\widetilde{T}_{0}=\max \left\{T_{1}, \widetilde{T}_{2}\right\}
$$

with

$$
T_{1}=\limsup _{k \longrightarrow+\infty} \frac{\log \left\|\psi_{1, k}\right\|_{H_{0}^{1}}}{k^{2}}, \quad \widetilde{T}_{2}=\limsup _{k \rightarrow+\infty} \frac{\log \frac{\left\|\psi_{2, k}-\psi_{1, i_{k}}\right\|}{\left|\nu k^{2}-i_{k}^{2}\right|}}{\nu k^{2}}=\limsup _{k \rightarrow+\infty} \frac{\log \frac{\left\|\psi_{2, k}-\psi_{1, i_{k}}\right\|}{\left|\nu k^{2}-i_{k}^{2}\right|}}{i_{k}^{2}}
$$

- Let us suppose $\widetilde{T}_{0}=T_{1}$, and work with the particular solutions of the adjoint problem (1.17) associated with initial data

$$
\theta_{k}^{0}=\psi_{1, k}
$$

where $\psi_{1, k}$ is given by (1.9). With this choice, the solution $\theta_{k}$ of the adjoint problem is given by

$$
\theta_{k}(x, t)=\psi_{1, k}(x) e^{-k^{2}(T-t)}
$$

The observability inequality reads as

$$
\left\|\theta_{k}(\cdot, 0)\right\|_{H_{0}^{1}}^{2} \leq C \int_{0}^{T}\left|B^{*} D \frac{\partial \theta_{k}(0, t)}{\partial x}(0, t)\right|^{2} \mathrm{~d} t
$$

From the definition of $\widetilde{T}_{1}$, there exists a sub-sequence $\left\{k_{n}\right\}_{n \geq 1}$ such that:

$$
\left.\left.\widetilde{T}_{1}=\lim _{n \rightarrow+\infty} \frac{\log \left\|\psi_{1, k_{n}}\right\|_{H_{0}^{1}}}{k_{n}^{2}} \in\right] 0,+\infty\right]
$$

in this case, for every $\varepsilon>0$, there exists a positive integer $n_{\varepsilon} \geq 1$ such that

$$
\left\|\psi_{1, k_{n}}\right\|_{H_{0}^{1}}>e^{k_{n}^{2}\left(T_{1}-\varepsilon\right)}, \quad \forall n \geq n_{\varepsilon},
$$

thus

$$
\left\{\begin{array}{l}
\left\|\theta_{k_{n}}(\cdot, 0)\right\|_{H_{0}^{1}}^{2}=e^{-2 k_{n}^{2} T}\left\|\psi_{1, k_{n}}\right\|_{H_{0}^{1}}^{2} \geq e^{-2 k_{n}^{2}\left[T-T_{1}+\varepsilon\right]} \underset{n \rightarrow+\infty}{\longrightarrow}+\infty \\
\int_{0}^{T}\left|B^{*} D \frac{\partial \theta_{k_{n}}(0, t)}{\partial x}(0, t)\right|^{2} \mathrm{~d} t=\int_{0}^{T} e^{-2 k_{n}^{2} t} \mathrm{~d} t \underset{n \rightarrow+\infty}{\longrightarrow} 0
\end{array}\right.
$$

This proves that the observability inequality does not hold

- Let us suppose now, $\widetilde{T}_{0}=\widetilde{T}_{2}$. Let us fix $k \geq 1$ and work with the particular solutions associated with initial data

$$
\theta_{k}^{0}=\psi_{1, i_{k}}-\psi_{2, k}
$$


with this choice, the solution $\theta_{k}$ of adjoint problem is given by

$$
\theta_{k}(x, t)=\psi_{1, i_{k}}(x) e^{-i_{k}^{2}(T-t)}-\psi_{2, k}(x) e^{-\nu k^{2}(T-t)}
$$

We have

$$
\left\{\begin{array}{l}
\left\|\theta_{k}(\cdot, 0)\right\|_{H_{0}^{1}}^{2}=\left\|e^{-i_{k}^{2} T}\left(\psi_{1, i_{k}}-\psi_{2, k}\right)+\psi_{2, k}\left(e^{-i_{k}^{2} T}-e^{-\nu k^{2} T}\right)\right\|_{H_{0}^{1}}^{2} \\
\int_{0}^{T}\left|B^{*} D \frac{\partial \theta_{k}(0, t)}{\partial x}(0, t)\right|^{2} \mathrm{~d} t=\int_{0}^{T}\left|e^{-i_{k}^{2} t}-e^{-\nu k^{2} t}\right|^{2} \mathrm{~d} t
\end{array}\right.
$$

thus we obtain

$$
\left\{\begin{array}{l}
\left\|\theta_{k}(\cdot, 0)\right\|_{H_{0}^{1}} \geq e^{-2 i_{k}^{2} T}\left\|\psi_{1, i_{k}}-\psi_{2, k}\right\|^{2}-2 e^{-i_{k}^{2} T}\left\|\psi_{1, i_{k}}-\psi_{2, k}\right\|\left\|\psi_{2, k}\right\|\left|i_{k}^{2}-\nu k^{2}\right| \\
\int_{0}^{T}\left|B^{*} D \frac{\partial \theta_{k}(0, t)}{\partial x}(0, t)\right|^{2} \mathrm{~d} t \leq\left|i_{k}^{2}-\nu k^{2}\right|^{2},
\end{array}\right.
$$

and

$$
\frac{\left\|\theta_{k}(\cdot, 0)\right\|_{H_{0}^{1}}^{2}}{\int_{0}^{T}\left|B^{*} D \frac{\partial \theta_{k}(0, t)}{\partial x}(0, t)\right|^{2} \mathrm{~d} t} \geq e^{-2 i_{k}^{2} T} \frac{\left\|\psi_{1, i_{k}}-\psi_{2, k}\right\|^{2}}{\left|i_{k}^{2}-\nu k^{2}\right|^{2}}\left(1-2 e^{i_{k}^{2} T} \frac{\left|i_{k}^{2}-\nu k^{2}\right|}{\left\|\psi_{1, i_{k}}-\psi_{2, k}\right\|}\left\|\psi_{2, i_{k}}\right\|\right) .
$$

From the definition of $\widetilde{T}_{2}$, there exists an increasing unbounded sub-sequence $\left(k_{n}\right)_{n \geq 1}$ such that

$$
\left.\left.\widetilde{T}_{2}=\lim _{n \rightarrow+\infty} \frac{\log \frac{\left\|\psi_{2, k_{n}}-\psi_{1, i_{k_{n}}}\right\|}{\left|\nu k_{n}^{2}-i_{k_{n}}^{2}\right|}}{i_{k_{n}^{2}}} \in\right] 0,+\infty\right]
$$

in this case, for every $\varepsilon>0$, there exists a positive integer $n_{\varepsilon} \geq 1$ such that

$$
\frac{\left\|\psi_{2, k_{n}}-\psi_{1, i_{k_{n}}}\right\|}{\left|\nu k_{n}^{2}-i_{k_{n}}^{2}\right|}>e^{i_{k_{n}}^{2}\left(\widetilde{T}_{2}-\varepsilon\right)}, \quad \forall n \geq n_{\varepsilon}
$$

thus

$$
e^{-i_{k_{n}}^{2} T} \frac{\left\|\psi_{2, k_{n}}-\psi_{1, i_{k_{n}}}\right\|}{\left|\nu k_{n}^{2}-i_{k_{n}}^{2}\right|}>e^{i_{k_{n}}^{2}\left(\widetilde{T}_{2}-T-\varepsilon\right)} \underset{n \rightarrow+\infty}{\longrightarrow}+\infty, \quad \forall \varepsilon \in\left(0, \widetilde{T}_{2}-T\right)
$$

This proves that the observability inequality does not hold and finishes the proof of the negative null controllability result of (1.1).

\subsection{Proof of Theorem 1.1: second case (b)}

In this subsection, our objective is to prove that system (1.1) is null controllable at time $T$ if $T>\widetilde{T}_{0} \in[0, \infty)$, when $\sqrt{\nu}<1$. Thus, in the sequel we consider $\sqrt{\nu}<1$. 


\subsubsection{Positive null controllability result}

Let us start with the following crucial result.

Proposition 2.10. Let $\sqrt{\nu}<1$. Let us define the function

$$
\begin{aligned}
J: \mathbb{N}^{*} & \longrightarrow \mathbb{N}^{*} \\
k & \longmapsto j_{k},
\end{aligned}
$$

where, for any $k \in \mathbb{N}^{*}, j_{k}$ is the nearest integer to $\frac{k}{\sqrt{\nu}}$ i.e $\left|\frac{k}{\sqrt{\nu}}-j_{k}\right|<\frac{1}{2}$. Thus for any $k \in \mathbb{N}^{*}$,

$$
\left|\frac{k}{\sqrt{\nu}}-j\right|>\frac{1}{2}, \quad \forall j \in \mathbb{N}^{*}, j \neq j_{k}
$$

Then

1. The function $J$ is injective.

2. $\widehat{J}=\mathbb{N}^{*} \backslash J\left(\mathbb{N}^{*}\right)=\left\{\widehat{j}_{k}: k \geq 1\right\}$ is a infinite set, where the elements of $\widehat{J}$ are classified in ascending order.

Proof of Proposition 2.10. The proof can be done in the same way as in Proposition 2.7.

Thus, thanks to Proposition 2.10 we can reformulate (2.13). We say that the null controllability property at time $T$ for system (1.1) is equivalent to find $u \in L^{2}(0, T)$ such that:

$$
\left\{\begin{array}{l}
\int_{0}^{T} e^{-k^{2} t} u(T-t) \mathrm{d} t=-e^{-k^{2} T}\left\langle y^{0}, \psi_{1, k}\right\rangle_{-1, i}, \\
\int_{0}^{T} e^{-\nu j_{k}^{2} t} u(T-t) \mathrm{d} t=-e^{-\nu j_{k}^{2} T}\left\langle y^{0}, \psi_{2, j_{k}}\right\rangle_{-1,1}, \\
\int_{0}^{T} e^{-\nu \widehat{j}_{k}^{2} t} u(T-t) \mathrm{d} t=-e^{-\nu \widehat{j}_{k}^{2} T}\left\langle y^{0}, \psi_{2, \widehat{j}_{k}}\right\rangle_{-1,1}, \quad k \geq 1 .
\end{array}\right.
$$

We can now state our following main result.

Proposition 2.11. There exists $\left\{q_{k}\right\}_{k \geq 1} \subset E_{T}$ such that

1.

$$
\left\{\begin{array}{l}
\int_{0}^{T} e^{-k^{2} t} q_{k}(t) \mathrm{d} t=-e^{-k^{2} T}\left\langle y^{0}, \psi_{1, k}\right\rangle_{-1, i}, \\
\int_{0}^{T} e^{-\nu j_{k}^{2} t} q_{k}(t) \mathrm{d} t=-e^{-\nu j_{k}^{2} T}\left\langle y^{0}, \psi_{1, j_{k}}\right\rangle_{-1,1}, \\
\int_{0}^{T} e^{-\nu \widehat{j}_{k}^{2} t} q_{k}(t) \mathrm{d} t=-e^{-\nu \widehat{j}_{k}^{2} T}\left\langle y^{0}, \psi_{2, \widehat{j}_{k}}\right\rangle_{-1,1}, \quad k \geq 1, \\
\int_{0}^{T} e^{-k^{2} t} q_{i}(t) \mathrm{d} t=\int_{0}^{T} e^{-\nu j_{k}^{2} t} q_{i}(t) \mathrm{d} t=\int_{0}^{T} e^{-\nu \widehat{j}_{k}^{2} t} q_{i}(t) \mathrm{d} t=0, k \neq i .
\end{array}\right.
$$


2. If $T>\widehat{T}_{0}=\max \left\{T_{1}, \widehat{T}_{2}\right\}$ (see (1.13) then we infer that an explicit solution $u$ of moment problem (2.26) given by

$$
u(t)=\sum_{k \geq 1} q_{k}(T-t)
$$

Proof of Proposition 2.11. Just use here the same reasoning as in the previous Proposition 2.8.

\subsubsection{The negative null controllability result}

Let us prove that if $0<T<\widehat{T}_{0}$, then system (1.1) is not null controllable at time $T$. We argue by contradiction. Let us first recall that

$$
\widehat{T}_{0}=\max \left\{T_{1}, \widehat{T}_{2}\right\}
$$

where

$$
T_{1}=\limsup _{k \rightarrow+\infty} \frac{\log \left\|\psi_{1, k}\right\|_{H_{0}^{1}}}{k^{2}} \text { and } \widehat{T}_{2}=\limsup _{k \rightarrow+\infty} \frac{\log \frac{\left\|\psi_{1, k}-\psi_{2, j_{k}}\right\|}{\left|k^{2}-\nu j_{k}^{2}\right|}}{k^{2}}=\limsup _{k \rightarrow+\infty} \frac{\log \frac{\left\|\psi_{1, k}-\psi_{1, j_{k}}\right\|}{\left|k^{2}-\nu j_{k}^{2}\right|}}{\nu j_{k}^{2}}
$$

Assume that system (1.1) is null-controllable at time $T<\widehat{T}_{0}$. System (1.1) is null-controllable at time $T$ if and only if there exists $C>0$ such that any solution $\theta$ of the adjoint problem (1.17) satisfies the observability inequality:

$$
\|\theta(0)\|_{H_{0}^{1}\left(0, \pi ; \mathbb{R}^{2}\right)}^{2} \leq C \int_{0}^{T}\left|B^{*} D \theta_{x}(0, t)\right|^{2} \mathrm{~d} t, \quad \forall \theta^{0} \in H_{0}^{1}\left(0, \pi ; \mathbb{R}^{2}\right) .
$$

- Let us suppose $\widehat{T}_{0}=T_{1}$ and work with the particular solutions of adjoint problem (1.17) associated with initial data

$$
\theta_{k}^{0}=\psi_{1, k}
$$

where $\psi_{1, k}$ is given by (1.9). With this choice, the solution $\theta_{k}$ of adjoint problem is given by

$$
\theta_{k}(x, t)=\psi_{1, k}(x) e^{-k^{2}(T-t)}
$$

The observability inequality reads as

$$
\left\|\theta_{k}(\cdot, 0)\right\|_{H_{0}^{1}}^{2} \leq C \int_{0}^{T}\left|B^{*} D \frac{\partial \theta_{k}(0, t)}{\partial x}(0, t)\right|^{2} \mathrm{~d} t
$$


From the definition of $T_{1}$, there exists a subsequence $\left\{k_{n}\right\}_{n \geq 1}$ such that:

$$
\left.\left.T_{1}=\lim _{n \rightarrow+\infty} \frac{\log \left\|\psi_{1, k_{n}}\right\|_{H_{0}^{1}}}{k_{n}^{2}} \in\right] 0,+\infty\right]
$$

in this case, for every $\varepsilon>0$, there exits a positive integer $n_{\varepsilon} \geq 1$ such that

$$
\left\|\psi_{1, k_{n}}\right\|_{H_{0}^{1}}>e^{k_{n}^{2}\left(T_{1}-\varepsilon\right)}, \quad \forall n \geq n_{\varepsilon},
$$

thus,

$$
\left\{\begin{array}{l}
\left\|\theta_{k_{n}}(\cdot, 0)\right\|_{H_{0}^{1}}^{2}=e^{-2 k_{n}^{2} T}\left\|\psi_{1, k_{n}}\right\|_{H_{0}^{1}}^{2} \geq e^{-2 k_{n}^{2}\left[T-T_{1}+\varepsilon\right]} \longrightarrow+\infty, \quad \text { when } n \longrightarrow+\infty \\
\int_{0}^{T}\left|B^{*} D \frac{\partial \theta_{k_{n}}(0, t)}{\partial x}(0, t)\right|^{2} \mathrm{~d} t=\int_{0}^{T} e^{-2 k_{n}^{2} t} \mathrm{~d} t \longrightarrow 0, \quad \text { when } n \longrightarrow+\infty
\end{array}\right.
$$

This proves that the observability inequality does not hold.

- Assume $\widehat{T}_{0}=\widehat{T}_{2}$. Let us fix $k \geq 1$ and work with the particular solutions associated with initial data

$$
\theta_{k}^{0}=\psi_{1, k}-\psi_{2, j_{k}}
$$

With this choice, the solution $\theta_{k}$ of adjoint problem is given by

$$
\theta_{k}(x, t)=\psi_{1, k}(x) e^{-k^{2}(T-t)}-\psi_{2, j_{k}}(x) e^{-\nu j_{k}^{2}(T-t)} .
$$

We have

$$
\left\{\begin{array}{l}
\left\|\theta_{k}(\cdot, 0)\right\|_{H_{0}^{1}}^{2}=\left\|e^{-k^{2} T}\left(\psi_{1, k}-\psi_{2, j_{k}}\right)+\psi_{2, j_{k}}\left(e^{-k^{2} T}-e^{-\nu j_{k}^{2} T}\right)\right\|_{H_{0}^{1}}^{2} \\
\int_{0}^{T}\left|B^{*} D \frac{\partial \theta_{k}(0, t)}{\partial x}(0, t)\right|^{2} \mathrm{~d} t=\int_{0}^{T}\left|e^{-k^{2} t}-e^{-\nu j_{k}^{2} t}\right|^{2} \mathrm{~d} t
\end{array}\right.
$$

Thus we obtain

$$
\left\{\begin{array}{l}
\left\|\theta_{k}(\cdot, 0)\right\|_{H_{0}^{1}} \geq e^{-2 k^{2} T}\left\|\psi_{1, k}-\psi_{2, j_{k}}\right\|^{2}-2 e^{-k^{2} T}\left\|\psi_{1, k}-\psi_{2, j_{k}}\right\|\left\|\psi_{2, j_{k}}\right\|\left|k^{2}-\nu j_{k}^{2}\right|, \\
\int_{0}^{T}\left|B^{*} D \frac{\partial \theta_{k}(0, t)}{\partial x}(0, t)\right|^{2} \mathrm{~d} t \leq\left|k^{2}-\nu j_{k}^{2}\right|^{2}
\end{array}\right.
$$

and

$$
\frac{\left\|\theta_{k}(\cdot, 0)\right\|_{H_{0}^{1}}^{2}}{\int_{0}^{T}\left|B^{*} D \frac{\partial \theta_{k}(0, t)}{\partial x}(0, t)\right|^{2} \mathrm{~d} t} \geq e^{-2 k^{2} T} \frac{\left\|\psi_{1, k}-\psi_{2, j_{k}}\right\|^{2}}{\left|k^{2}-\nu j_{k}^{2}\right|^{2}}\left(1-2 e^{k^{2} T} \frac{\left|k^{2}-\nu j_{k}^{2}\right|}{\left\|\psi_{1, k}-\psi_{2, j_{k}}\right\|}\left\|\psi_{2, j_{k}}\right\|\right) .
$$

From the definition of $\widehat{T}_{2}$, there exists an increasing unbounded subsequence $\left(k_{n}\right)_{n \geq 1}$ such that

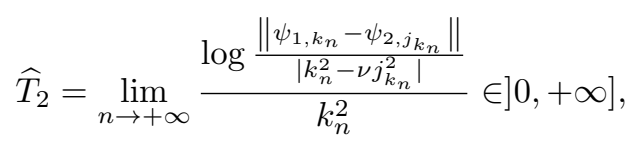


in this case, for every $\varepsilon>0$, there exits a positive integer $n_{\varepsilon} \geq 1$ such that

$$
\frac{\left\|\psi_{1, k_{n}}-\psi_{2, j_{n}}\right\|}{\left|k_{n}^{2}-\nu j_{k_{n}}^{2}\right|}>e^{k_{n}^{2}\left(\widehat{T}_{2}-\varepsilon\right)}, \quad \forall n \geq n_{\varepsilon}
$$

thus,

$$
e^{-k_{n}^{2} T} \frac{\left\|\psi_{2, k_{n}}-\psi_{1, i_{k_{n}}}\right\|}{\left|\nu k_{n}^{2}-i_{k_{n}}^{2}\right|}>e^{k_{n}^{2}\left(\widehat{T}_{2}-T-\varepsilon\right)} \longrightarrow+\infty, \quad \forall \varepsilon \in\left(0, \widehat{T}_{2}-T\right) .
$$

This proves that the observability inequality does not hold and finishes the proof of the negative null controllability result of (1.1).

\section{Null-CONTROllability RESUlt With AN RATIONAL DifFUSiON COEFFICIENT}

\subsection{Some preliminary results}

In this subsection we will give some properties which will be used below. We are interested in studying the spectrum of the operators $L^{*}$. Let us define the sets

$$
\left\{\begin{array}{l}
\Lambda_{1}:=\left\{\frac{i_{0}^{2}}{j_{0}^{2}} j^{2}: j \in \mathbb{N}^{*} \backslash j_{0} \mathbb{N}^{*}\right\} \\
\Lambda_{2}:=\left\{k^{2}: k \in \mathbb{N}^{*} \backslash i_{0} \mathbb{N}^{*}\right\} \\
\Lambda_{3}:=\left\{i_{0}^{2} l^{2}: l \geq 1\right\}
\end{array}\right.
$$

Observe that $\Lambda_{1}, \Lambda_{2}$ and $\Lambda_{3}$ are disjoint sets.

Remark 3.1. If $i_{0}=1$ then $\Lambda_{2}=\emptyset, \Lambda_{1}:=\left\{\frac{j^{2}}{j_{0}^{2}}: j \in \mathbb{N}^{*} \backslash j_{0} \mathbb{N}^{*}\right\}$ and $\Lambda_{3}:=\left\{l^{2}: l \geq 1\right\}$. Thus in the sequel, the controllability of the system (1.1) will be studied in only when $\sqrt{\nu}=\frac{i_{0}}{j_{0}} \in \mathbb{Q}_{+}^{*}$ and $i_{0}>1$. The case $i_{0}=1$ is much easier and it is left to the reader.

In the sequel, for the proof of Theorem 1.3, we shall use the following notations

$$
I(\zeta)=\int_{0}^{\pi} q(s) \varphi_{\sqrt{\zeta}}(s) \sqrt{\frac{\pi}{2}} \sin \left(\sqrt{\frac{\zeta}{\nu}}(\pi-s)\right) \mathrm{d} s, \quad \zeta \in \Lambda_{2} \cup \Lambda_{3}=\left\{k^{2}: k \geq 1\right\},
$$

and $T_{0}(\nu, q)$ becomes

$$
T_{0}(\nu, q):=\limsup _{\zeta \in \Lambda_{2} \cup \Lambda_{3}, \zeta \rightarrow+\infty} \frac{-\log |I(\zeta)|}{\zeta} \in[0,+\infty]
$$

Proposition 3.2. Let $A_{0}$ be given by (1.2) and consider the operator $(L, D(L))$ given by (1.6) and its adjoint $L^{*}$.

1. The spectrum of $L^{*}$ is given by

$$
\sigma\left(L^{*}\right)=\Lambda_{1} \cup \Lambda_{2} \cup \Lambda_{3} .
$$


2. Given $j \in \mathbb{N}^{*} \backslash j_{0} \mathbb{N}^{*}, k \in \mathbb{N}^{*} \backslash i_{0} \mathbb{N}^{*}$ and $l \geq 1$, let us introduce

$$
\Phi_{2, j}^{*}:=\left(\begin{array}{c}
0 \\
\varphi_{j}
\end{array}\right), \widetilde{\Phi}_{1, k}^{*}:=\left(\begin{array}{c}
\varphi_{k} \\
\widetilde{\psi}_{k}
\end{array}\right) \text { and } \widehat{\Phi}_{1, l}^{*}:=\left(\begin{array}{c}
\varphi_{i_{0} l} \\
\widehat{\psi}_{l}
\end{array}\right) .
$$

(a) Given $j \in \mathbb{N}^{*} \backslash j_{0} \mathbb{N}^{*}$, then

$$
\left(L^{*}-\nu j^{2} I_{d}\right) \Phi_{2, j}^{*}=0 .
$$

(b) Given $k \in \mathbb{N}^{*} \backslash i_{0} \mathbb{N}^{*}$, then

$$
\left(L^{*}-k^{2} I_{d}\right) \widetilde{\Phi}_{1, k}^{*}=0 .
$$

where, $\widetilde{\psi}_{k}$ is the unique solution of following problem:

$$
\left\{\begin{array}{l}
-\widetilde{\psi}_{k}-\frac{k^{2}}{\nu} \widetilde{\psi}_{k}=-\frac{1}{\nu} q \varphi_{k} \quad \text { in }(0, \pi) \\
\widetilde{\psi}_{k}(0)=\widetilde{\psi}_{k}(\pi)=0
\end{array}\right.
$$

Moreover, an explicit expression of $\widetilde{\psi}_{k}$ is given by:

$$
\widetilde{\psi}_{k}(x)=\widetilde{\psi}_{k}^{\prime}(0) \frac{\sqrt{\nu}}{k} \sin \left(\frac{k x}{\sqrt{\nu}}\right)+\frac{\sqrt{\nu}}{\nu k} \int_{0}^{x} \sin \left(\frac{k}{\sqrt{\nu}}(x-\xi)\right) q(\xi) \varphi_{k}(\xi) \mathrm{d} \xi,
$$

with (see Rem. 2.2)

$$
\widetilde{\psi}_{k}^{\prime}(0)=-\frac{I\left(k^{2}\right)}{\nu \sqrt{\frac{\pi}{2}} \sin \left(\frac{k \pi}{\sqrt{\nu}}\right)}
$$

(c) Given $l \geq 1$, then

$$
\left\{\begin{array}{l}
\left(L^{*}-i_{0}^{2} l^{2} I_{d}\right) \Phi_{2, j_{0} l}^{*}=0, \\
\left(L^{*}-i_{0}^{2} l^{2} I_{d}\right) \widehat{\Phi}_{1, l}^{*}=I\left(i_{0}^{2} l^{2}\right) \Phi_{2, j_{0} l}^{*}, \quad \forall l \geq 1,
\end{array}\right.
$$

and $\widehat{\psi}_{l}$ is the unique solution of following problem:

$$
\left\{\begin{array}{l}
-\widehat{\psi}_{l}-j_{0}^{2} l^{2} \widehat{\psi}_{l}=\frac{1}{\nu}\left[I\left(i_{0}^{2} l^{2}\right) \varphi_{j_{0} l}-q \varphi_{i_{0} l}\right] \quad \text { in }(0, \pi), \\
\widehat{\psi}_{l}(0)=\widehat{\psi}_{l}(\pi)=0 \\
\int_{0}^{\pi} \widehat{\psi}_{l}(x) \varphi_{j_{0} l}(x) \mathrm{d} x=0,
\end{array}\right.
$$


an explicit expression of $\widehat{\psi}_{l}$ is given by:

$$
\widehat{\psi}_{l}(x)=\alpha_{l} \varphi_{j_{0} l}(x)+\beta_{l}(x), \quad \text { for all } \quad x \in(0, \pi),
$$

where

$$
\left\{\begin{aligned}
\beta_{l}(x) & =-\frac{1}{\nu j_{0} l} \int_{0}^{x} \sin \left(j_{0} l(x-\xi)\right)\left[I\left(i_{0}^{2} l^{2}\right) \varphi_{j_{0} l}(\xi)-q(\xi) \varphi_{i_{0} l}(\xi)\right] \mathrm{d} \xi \\
\alpha_{l} & =\frac{1}{\nu j_{0} l} \int_{0}^{\pi} \int_{0}^{x} \sin \left(j_{0} l(x-\xi)\right)\left[I\left(i_{0}^{2} l^{2}\right) \varphi_{j_{0} l}(\xi)-q(\xi) \varphi_{i_{0} l}(\xi)\right] \varphi_{j_{0} l}(x) \mathrm{d} \xi \mathrm{d} x
\end{aligned}\right.
$$

Moreover (see Rem. 2.2 for instance)

$$
\beta_{l}^{\prime}(x)=-\frac{1}{\nu} \int_{0}^{x} \cos \left(j_{0} l(x-\xi)\right)\left[I\left(i_{0}^{2} l^{2}\right) \varphi_{j_{0} l}(\xi)-q(\xi) \varphi_{i_{0} l}(\xi)\right] \mathrm{d} \xi
$$

thus $\beta_{l}^{\prime}(0)=0$.

In the next result we are going to give some properties of $\widetilde{\psi}_{k}$ (see 3.6) and $\widehat{\psi}_{l}$ (see 3.8). These properties will be used later and will be crucial in the proof of Theorem 1.3.

Proposition 3.3. 1. Let us fix $q \in L^{\infty}(0, \pi)$ and take $k \in \mathbb{N}^{*} \backslash i_{0} \mathbb{N}^{*}$. Then, one has:

$$
\widetilde{\psi}_{k}^{\prime}(x)=-\frac{I\left(k^{2}\right)}{\nu \sqrt{\frac{\pi}{2}} \sin \left(\frac{k \pi}{\sqrt{\nu}}\right)} \cos \left(\frac{k x}{\sqrt{\nu}}\right)+\frac{1}{\nu} \int_{0}^{x} \cos \left(\frac{k}{\sqrt{\nu}}(x-\xi)\right) q(\xi) \varphi_{k}(\xi) \mathrm{d} \xi
$$

In addition, there exists a constant $C>0$ such that

$$
\left\|\widetilde{\psi}_{k}^{\prime}\right\|_{L^{\infty}(0, \pi)} \leq C, \quad k \in \mathbb{N}^{*} \backslash i_{0} \mathbb{N}^{*}
$$

2. Let us take $l \geq 1$. There exists a constant $C>0$ such that

$$
\left|\alpha_{l}\right| \leq C \quad \text { and } \quad\left\|\widehat{\psi}_{l}^{\prime}\right\|_{L^{\infty}(0, \pi)} \leq C, \quad l \geq 1
$$

Proof of Proposition 3.3. 
1. Let us fix $k \in \mathbb{N}^{*} \backslash i_{0} \mathbb{N}^{*}$, the expression (3.10) can be deduced from (2.5) and (2.6). Moreover, there exists a constant $C>0$ such that

$$
\begin{aligned}
\left\|\widetilde{\psi}_{k}^{\prime}\right\|_{L^{\infty}(0, \pi)} \leq & \frac{C}{\left|\sin \left(\frac{k \pi}{\sqrt{\nu}}\right)\right|}+C, \\
& =\frac{C}{\left|\sin \left(\pi\left(n_{k}-\frac{k j_{0}}{i_{0}}\right)\right)\right|}+C \quad\left(\text { for each } k, n_{k} \text { is the nearest integer of } \frac{k j_{0}}{i_{0}}\right), \\
& \leq \frac{\pi C}{2\left|\pi\left(n_{k}-\frac{k j_{0}}{i_{0}}\right)\right|}+C \quad\left(0<\left|n_{k}-\frac{k j_{0}}{i_{0}}\right| \leq \frac{1}{2}, \forall x \in\left[0, \frac{\pi}{2}\right], \frac{2}{\pi} x \leq \sin x\right), \\
& \leq \frac{i_{0} C}{2}+C \quad\left(\text { since } 0<\frac{1}{i_{0}} \leq\left|n_{k}-\frac{k j_{0}}{i_{0}}\right| \leq \frac{1}{2}\right) .
\end{aligned}
$$

2. The properties (3.11) can be deduced from (3.8) . This finalizes the proof.

Proof of Proposition 3.2.

First, let $\lambda$ be an eigenvalue of $L^{*}$ and $y=\left(y_{1}, y_{2}\right)^{T}$ an associated eigenfunction. Thus $y$ is a solution of the following problem:

$$
\begin{cases}-y_{1}^{\prime \prime}=\lambda y_{1} & \text { in }(0, \pi) \\ q y_{1}-\nu y_{2}^{\prime \prime}=\lambda y_{2} & \text { in }(0, \pi) \\ y_{1}(0)=y_{2}(0)=0, \quad y_{1}(\pi)=y_{2}(\pi)=0 . & \end{cases}
$$

- If $y_{1} \equiv 0$, then, $\lambda=\nu j^{2}$ is an eigenvalue of $L^{*}$ and taking $y_{2}=\varphi_{j}$, we obtain $\Phi_{2, j}^{*}$ as associated eigenfunction of $L^{*}$.

- Now assume that $y_{1} \not \equiv 0$, then $\lambda=k^{2}$ and $y_{1}=\varphi_{k}$ is a solution to the first o.d.e. Inserting this expression in the second equation, we get for $y_{2}$ :

$$
\left\{\begin{array}{l}
y_{2}^{\prime \prime}+\frac{k^{2}}{\nu} y_{2}=\frac{1}{\nu} q \varphi_{k} \text { in }(0, \pi) \\
y_{2}(0)=y_{2}(\pi)=0
\end{array}\right.
$$

Let us multiply $(3.12)$ by $\sin \left(\frac{k}{\sqrt{\nu}}(\pi-s)\right)$ and using partial integration, we obtain:

$$
\begin{cases}y_{2}^{\prime}(0)=\frac{-\int_{0}^{\pi} q(s) \varphi_{k}(s) \sin \left(\frac{k}{\sqrt{\nu}}(\pi-s)\right) \mathrm{d} s}{\nu \sin \left(\frac{k \pi}{\sqrt{\nu}}\right)}=-\frac{I\left(k^{2}\right)}{\nu \sqrt{\frac{\pi}{2}} \sin \left(\frac{k \pi}{\sqrt{\nu}}\right)}, & \text { if } k \neq i_{0} l \\ \int_{0}^{\pi} q(s) \varphi_{i_{0} l}(s) \varphi_{j_{0} l}(s) \mathrm{d} s=I\left(i_{0}^{2} l^{2}\right)=0, & \text { if } k=i_{0} l .\end{cases}
$$

○ Observe that, suppose $k=i_{0} l$, thus (3.12) admits a solution if and only if $I\left(i_{0}^{2} l^{2}\right)=0$. In this case, $i_{0}^{2} l^{2}$ is a double eigenvalue of $L^{*}$ (take $j=j_{0} l$ ). From the above considerations, it is clear that if $I\left(i_{0}^{2} l^{2}\right) \neq 0$, then the eigenvalue $i_{0}^{2} l^{2}$ of $L^{*}$ is simple and $\Phi_{2, j_{0} l}^{*}$ is an associated eigenfunction. Observe that, taking 
$\widehat{\Phi}_{1, l}^{*}=\left(z_{1}, z_{2}\right)^{T}$ the equation $\left(L^{*}-i_{0}^{2} l^{2} I_{d}\right) \widehat{\Phi}_{1, l}^{*}=c \Phi_{2, j_{0} l}$ writes:

$$
\begin{cases}-z_{1}^{\prime \prime}-i_{0}^{2} l^{2} z_{1}=0 & \text { in }(0, \pi), \\ -\nu z_{2}^{\prime \prime}-i_{0}^{2} l^{2} z_{2}=\left[c \varphi_{j_{0} l}-q z_{1}\right] & \text { in }(0, \pi), \\ z_{1}(0)=z_{2}(0)=0, \quad z_{1}(\pi)=z_{2}(\pi)=0 . & \end{cases}
$$

Thus, choosing $z_{1}=\varphi_{i_{0} l}$ and inserting this expression in the second equation, we get:

$$
\left\{\begin{array}{l}
-z_{2}^{\prime \prime}-j_{0}^{2} l^{2} z_{2}=\frac{1}{\nu}\left[c \varphi_{j_{0} l}-q \varphi_{i_{0} l}\right] \quad \text { in }(0, \pi), \\
z_{2}(0)=z_{2}(\pi)=0
\end{array}\right.
$$

A necessary and sufficient condition for the previous no-homogeneous Sturm-Liouville problem to have a solution is that

$$
\int_{0}^{\pi}\left[c \varphi_{j_{0} l}^{2}(x)-q(x) \varphi_{i_{0} l}(x) \varphi_{j_{0} l}(x)\right] \mathrm{d} x=0, \quad \text { i.e } \quad c=\int_{0}^{\pi} q \varphi_{i_{0} l} \varphi_{j_{0} l} \mathrm{~d} s .
$$

With this value of $\mathrm{c},(3.13)$ has a continuum of solutions. This proves that, for $k=i_{0} l, \widehat{\Phi}_{1, l}$ is a generalized eigenfunction of $L^{*}$ associated to $\lambda=i_{0}^{2} l^{2}$.

$\circ$ Otherwise, if $k \neq i_{0} l$, then

$$
y_{2}^{\prime}(0)=-\frac{\int_{0}^{\pi} q(s) \varphi_{k}(s) \sin \left(\frac{k}{\sqrt{\nu}}(\pi-s)\right) \mathrm{d} s}{\nu \sin \left(\frac{k \pi}{\sqrt{\nu}}\right)}=-\frac{I\left(k^{2}\right)}{\nu \sqrt{\frac{\pi}{2}} \sin \left(\frac{k \pi}{\sqrt{\nu}}\right)},
$$

and $\widetilde{\Phi}_{1, k}$ is a eigenfunction of $L^{*}$ associated to $\lambda=k^{2}$ with $k \neq i_{0} l$.

Lemma 3.4. The family $\mathcal{B}^{*}=\left\{\Phi_{2, j}^{*}: j \in \mathbb{N}^{*} \backslash j_{0} \mathbb{N}^{*} ; \widetilde{\Phi}_{1, k}^{*}: k \in \mathbb{N}^{*} \backslash i_{0} \mathbb{N}^{*} ; \Phi_{2, j_{0} l}^{*}, \widehat{\Phi}_{1, l}^{*}: l \geq 1\right\}$ is complete in $L^{2}\left(0, \pi ; \mathbb{R}^{2}\right)$.

Proof of Lemma 3.4. Indeed, if $f=\left(f_{1}, f_{2}\right)$ is such that

$$
\begin{cases}\left\langle f, \Phi_{2, j}^{*}\right\rangle=0, & j \in \mathbb{N}^{*} \backslash j_{0} \mathbb{N}^{*} \\ \left\langle f, \widetilde{\Phi}_{1, k}^{*}\right\rangle=0, & k \in \mathbb{N}^{*} \backslash i_{0} \mathbb{N}^{*} \\ \left\langle f, \Phi_{2, j_{0} l}^{*}\right\rangle=0, & l \geq 1 \\ \left\langle f, \widehat{\Phi}_{1, l}^{*}\right\rangle=0, & l \geq 1 .\end{cases}
$$

Then in particular

$$
\begin{cases}\left\langle f_{2}, \varphi_{j}\right\rangle=0, & j \in \mathbb{N}^{*} \backslash j_{0} \mathbb{N}^{*}, \\ \left\langle f_{1}, \varphi_{k}\right\rangle+\left\langle f_{2}, \widetilde{\psi}_{k}\right\rangle=0, & k \in \mathbb{N}^{*} \backslash i_{0} \mathbb{N}^{*}, \\ \left\langle f_{2}, \varphi_{j_{0}}\right\rangle=0, & l \geq 1, \\ \left\langle f_{1}, \varphi_{i_{0} l}\right\rangle+\left\langle f_{2}, \widehat{\psi}_{l}\right\rangle=0, & l \geq 1 .\end{cases}
$$


This implies that $f_{1}=f_{2}=0$ (since $\left\{\varphi_{k}\right\}_{k \geq 1}$ is an orthonormal basis in $L^{2}(0, \pi)$ ) and proves the completeness of $\mathcal{B}^{*}$.

\subsection{Proof of Theorem 1.3}

\subsubsection{Approximate controllability}

This subsection is devoted to proving the approximate controllability of system (1.1), that is to say, the first point of Theorem 1.3. To this end, we are going to apply the property (2.11).

Necessary condition: Let us suppose that condition (1.15) does not hold i.e. there exists $k_{0} \in \mathbb{N}^{*} \backslash i_{0} \mathbb{N}^{*}$ (resp. $\left.l_{0} \in \mathbb{N}^{*}\right)$ such that $I\left(k_{0}^{2}\right)=0\left(\right.$ resp. $\left.I\left(i_{0}^{2} l_{0}^{2}\right)=0\right)$.

- If $I\left(k_{0}^{2}\right)=0$, with $k_{0} \in \mathbb{N}^{*} \backslash i_{0} \mathbb{N}^{*}$, then we deduce that

$$
\widetilde{\Phi}_{1, k_{0}}^{*}=\left(\begin{array}{c}
\varphi_{k_{0}} \\
\widetilde{\psi}_{k_{0}}
\end{array}\right) \quad(\text { see }(3.4))
$$

is an non-trivial eigenfunction associated with the eigenvalue $k_{0}^{2}$ of the operator $L^{*}$ satisfying

$$
B^{*} D \frac{\partial \widetilde{\Phi}_{1, k_{0}}^{*}}{\partial x}(0)=\nu \widetilde{\psi}_{k_{0}}^{\prime}(0)=-\frac{I\left(k_{0}^{2}\right)}{\sqrt{\frac{\pi}{2}} \sin \left(\frac{k_{0} \pi}{\sqrt{\nu}}\right)}=0 \quad(\text { see }(3.7))
$$

- If $I\left(i_{0}^{2} l_{0}^{2}\right)=0$, with $l_{0} \in \mathbb{N}^{*}$, then we deduce that

$$
\widehat{\Phi}_{1, l_{0}}^{*}-\alpha_{l_{0}} \Phi_{2, j_{0} l_{0}}^{*}=\left(\begin{array}{c}
\varphi_{i_{0} l_{0}} \\
\beta_{l_{0}}
\end{array}\right) \quad(\text { see }(3.4) \text { and }(3.8)),
$$

is an non-trivial eigenfunction associated with the eigenvalue $i_{0}^{2} l_{0}^{2}$ of the operator $L^{*}$ satisfying

$$
B^{*} D \frac{\partial\left(\widehat{\Phi}_{1, l_{0}}^{*}-\alpha_{l_{0}} \Phi_{2, j_{0} l}^{*}\right)}{\partial x}(0)=\nu \beta_{l_{0}}^{\prime}(0)=0 \quad(\text { see }(3.9)) .
$$

Thus, system (1.1) is not approximately controllable at time $T$.

Sufficient condition: Let us suppose that condition (1.15) hold. The set of the eigenvectors associated with the eigenvalue $\nu k^{2}$ of $L^{*}$ is generated by $\Phi_{2, k}^{*}$ (see Prop. 2.1). In this case, we remark that for all $k \in \mathbb{N}^{*}$

$$
B^{*} D \frac{\partial \Phi_{2, k}^{*}}{\partial x}(0)=\nu \varphi_{k}^{\prime}(0)=k \nu \sqrt{\frac{2}{\pi}} \neq 0
$$

Moreover, the set of the eigenvectors associated with the eigenvalue $k^{2}, k \in \mathbb{N}^{*} \backslash i_{0} \mathbb{N}^{*}$, of $L^{*}$ is generated by $\widetilde{\Phi}_{1, k}^{*}$ (see Prop. 2.1). In this case, we remark that for all $k \in \mathbb{N}^{*} \backslash i_{0} \mathbb{N}^{*}$

$$
B^{*} D \frac{\partial \widetilde{\Phi}_{1, k}^{*}}{\partial x}(0)=\nu \widetilde{\psi}_{k}^{\prime}(0)=-\frac{I\left(k^{2}\right)}{\sqrt{\frac{\pi}{2}} \sin \left(\frac{k \pi}{\sqrt{\nu}}\right)} \neq 0 \quad(\text { see }(3.7)) .
$$

We conclude with the help of (2.11). 


\subsubsection{Positive null controllability result}

In this subsection, our objective is to prove that system (1.1) is exactly controllable to zero at time $T$ if $T>T_{0} \in[0, \infty)$ (see (1.16)). To this end, for $y_{0} \in H^{-1}\left(0, \pi ; \mathbb{R}^{2}\right)$ we will reformulate the null controllability problem as a moment problem. Let us first observe that condition (1.15) is a necessary condition for having the null controllability property of system (1.1) at time $T>0$. Using Proposition 1.5 and Proposition 1.6 we deduce that the control $u \in L^{2}(0, T)$ drives the solution of (1.1) to zero at time $T$ if and only if $u \in L^{2}(0, T)$ satisfies

$$
\int_{0}^{T} u(t) B^{*} D \theta_{x}(0, t) \mathrm{d} t=-\left\langle y^{0}, \theta(\cdot, 0)\right\rangle_{H^{-1}, H_{0}^{1}}, \quad \forall \theta^{0} \in H_{0}^{1}\left(0, \pi ; \mathbb{R}^{2}\right)
$$

where $\theta$ is the solution to the adjoint problem (1.17) associated with $\theta_{0}$. Since $\mathcal{B}^{*}$ is complete in $H_{0}^{1}\left(0, \pi ; \mathbb{R}^{2}\right)$, the null controllability property at time $T$ for system (1.1) is equivalent to find $u \in L^{2}(0, T)$ such that

$$
\begin{cases}\int_{0}^{T} u(t) B^{*} D \theta_{x}^{2, j}(0, t) \mathrm{d} t=-\left\langle y^{0}, \theta^{2, j}(\cdot, 0)\right\rangle_{H^{-1}, H_{0}^{1}}, & j \in \mathbb{N}^{*} \backslash j_{0} \mathbb{N}^{*}, \\ \int_{0}^{T} u(t) B^{*} D \widetilde{\theta}_{x}^{1, k}(0, t) \mathrm{d} t=-\left\langle y^{0}, \widetilde{\theta}^{1, k}(\cdot, 0)\right\rangle_{H^{-1}, H_{0}^{1}}, & k \in \mathbb{N}^{*} \backslash i_{0} \mathbb{N}^{*}, \\ \int_{0}^{T} u(t) B^{*} D \theta_{x}^{2, j_{0} l}(0, t) \mathrm{d} t=-\left\langle y^{0}, \theta^{2, j_{0} l}(\cdot, 0)\right\rangle_{H^{-1}, H_{0}^{1}}, & l \geq 1, \\ \int_{0}^{T} u(t) B^{*} D \widehat{\theta}_{x}^{1, l}(0, t) \mathrm{d} t=-\left\langle y^{0}, \widehat{\theta}^{1, l}(\cdot, 0)\right\rangle_{H^{-1}, H_{0}^{1}}, & l \geq 1,\end{cases}
$$

where $\theta^{2, j}$ is the solution of adjoint problem (1.17) associated with $\theta^{0}=\Phi_{2, j}^{*}$, and $\widetilde{\theta}^{1, k}$ (resp. $\widehat{\theta}^{1, l}$ ) is the solution of adjoint problem(1.17) associated with $\theta^{0}=\widetilde{\Phi}_{1, k}^{*}\left(\operatorname{resp} . \theta^{0}=\widehat{\Phi}_{1, k}^{*}\right)$. One has:

1. If we take $\theta^{0} \equiv \Phi_{2, j}^{*}$, the solution of the adjoint problem is $\theta^{2, j}(\cdot, t)=e^{-\nu j^{2}(T-t)} \Phi_{2, j}^{*}$ and we obtain:

$$
\int_{0}^{T} e^{-\nu j^{2} t} u(T-t) \mathrm{d} t=e^{-\nu j^{2} T}\left\langle y^{0}, \frac{\Phi_{2, j}^{*}}{\nu \varphi_{j}^{\prime}(0)}\right\rangle_{-1,1}=e^{-\nu j^{2} T} M\left(y^{0}, \nu j^{2}\right), \forall j \in \mathbb{N}^{*} \backslash j_{0} \mathbb{N}^{*},
$$

where

$$
M\left(y^{0}, \nu j^{2}\right)=\left\langle y^{0}, \frac{\Phi_{2, j}^{*}}{\nu \varphi_{j}^{\prime}(0)}\right\rangle_{H^{-1}, H_{0}^{1}}, \forall j \in \mathbb{N}^{*} \backslash j_{0} \mathbb{N}^{*} .
$$

Moreover

$$
\left|M\left(y^{0}, \alpha\right)\right| \leq C\left\|y_{0}\right\|_{H_{0}^{1}\left(0, \pi ; \mathbb{R}^{2}\right)}, \quad \forall \alpha \in \Lambda_{1} \quad(\text { see }(3.1)),
$$

for a positive constant $C$ independent of $\alpha \in \Lambda_{1}$ and $y^{0}$.

2. If we take $\theta^{0} \equiv \widetilde{\Phi}_{1, k}^{*}$, the solution of the adjoint problem is $\widetilde{\theta}^{1, k}(\cdot, t)=e^{-k^{2}(T-t)} \widetilde{\Phi}_{1, k}^{*}$ and we obtain:

$$
\int_{0}^{T} e^{-k^{2} t} u(T-t) \mathrm{d} t=e^{-k^{2} T}\left\langle y^{0}, \frac{\widetilde{\Phi}_{1, k}^{*}}{\nu \widetilde{\psi}_{k}^{\prime}(0)}\right\rangle_{-1,1}=\frac{e^{-k^{2} T}}{I\left(k^{2}\right)} \widetilde{M}\left(y^{0}, k^{2}\right), \forall k \in \mathbb{N}^{*} \backslash i_{0} \mathbb{N}^{*}
$$


where

$$
\widetilde{M}\left(y^{0}, k^{2}\right)=\nu \sqrt{\pi / 2} \sin \left(\frac{k \pi}{\sqrt{\nu}}\right)\left\langle y^{0}, \widetilde{\Phi}_{1, k}^{*}\right\rangle_{H^{-1}, H_{0}^{1}}, \quad \forall k \in \mathbb{N}^{*} \backslash i_{0} \mathbb{N}^{*} .
$$

Using the properties of the function $\widetilde{\psi}_{k}^{\prime}$ stated in Proposition 3.3, one has

$$
\left|\widetilde{M}\left(y^{0}, \beta\right)\right| \leq C\left\|y^{0}\right\|_{H_{0}^{1}\left(0, \pi ; \mathbb{R}^{2}\right)}, \quad \forall \beta \in \Lambda_{2},
$$

for a new positive constant $C$ independent of $\beta \in \Lambda_{2}$ and $y^{0}$.

3. Let us now take $\theta^{0} \equiv \Phi_{2, j_{0} l}^{*}\left(\right.$ resp. $\left.\theta^{0} \equiv \widehat{\Phi}_{1, l}^{*}\right)$, the solution of the adjoint problem is $\theta^{2, j_{0} l}(\cdot, t)=e^{-i_{0}^{2} l^{2}(T-t)} \Phi_{2, j_{0} l}^{*}\left(\operatorname{resp} . \widehat{\theta}^{1, l}(\cdot, t)=e^{-i_{0}^{2} l^{2}(T-t)}\left[\widehat{\Phi}_{1, l}^{*}-(T-t) I\left(i_{0}^{2} l^{2}\right) \Phi_{2, j_{0} l}^{*}\right]\right)$, for all $l \geq 1$. Thus, the control $u$ must also satisfy

$$
\left\{\begin{array}{l}
\int_{0}^{T} e^{-i_{0}^{2} l^{2} t} u(T-t) \mathrm{d} t=e^{-i_{0}^{2} l^{2} T} M^{*}\left(y^{0}, i_{0}^{2} l^{2}\right), \\
\int_{0}^{T} t e^{-i_{0}^{2} l^{2} t} u(T-t) \mathrm{d} t=\frac{e^{-i_{0}^{2} l^{2} T}}{I\left(i_{0}^{2} l^{2}\right)} \widehat{M}\left(y^{0}, i_{0}^{2} l^{2}\right),
\end{array} \quad \forall l \geq 1,\right.
$$

where

$$
M^{*}\left(y^{0}, i_{0}^{2} l^{2}\right)=\left\langle y^{0}, \frac{\Phi_{2, j_{0} l}^{*}}{\nu \varphi_{j_{0} l}^{\prime}(0)}\right\rangle_{H^{-1}, H_{0}^{1}}, \quad \forall l \geq 1
$$

and, for all $l \geq 1$

$$
\widehat{M}\left(y^{0}, i_{0}^{2} l^{2}\right)=\frac{1}{\nu \varphi_{j_{0} l}^{\prime}(0)}\left\langle y^{0}, \widehat{\Phi}_{1, l}^{*}+\left(\frac{\widehat{\psi}_{l}^{\prime}(0)}{\varphi_{j_{0} l}^{\prime}(0)}-T I\left(i_{0}^{2} l^{2}\right)\right) \Phi_{2, j_{0} l}^{*}\right\rangle_{-1,1} .
$$

Using the properties of the function $\widehat{\psi}_{k}^{\prime}$ stated in Proposition 3.3, one has

$$
\begin{cases}\left|\widehat{M}\left(y^{0}, \gamma\right)\right| \leq C\left\|y^{0}\right\|_{H_{0}^{1}\left(0, \pi ; \mathbb{R}^{2}\right)}, & \forall \gamma \in \Lambda_{3}, \\ \left|M^{*}\left(y^{0}, \gamma\right)\right| \leq C\left\|y^{0}\right\|_{H_{0}^{1}\left(0, \pi ; \mathbb{R}^{2}\right)}, & \forall \gamma \in \Lambda_{3}\end{cases}
$$

for a new positive constant $C$ independent of $\gamma \in \Lambda_{3}$ and $y^{0}$. 
Summarizing, we have proved that $u \in L^{2}(0 ; T)$ is such that the solution $y$ of system (1.1) satisfies $y(\cdot, T)=0$ in $(0 ; \pi)$ if and only if, $u \in L^{2}(0 ; T)$ satifies

$$
\begin{cases}\int_{0}^{T} e^{-\alpha t} u(T-t) \mathrm{d} t=e^{-\alpha T} M\left(y^{0}, \alpha\right), & \forall \alpha \in \Lambda_{1}, \\ \int_{0}^{T} e^{-\beta t} u(T-t) \mathrm{d} t=\frac{e^{-\beta T}}{I(\beta)} \widetilde{M}\left(y^{0}, \beta\right), & \forall \beta \in \Lambda_{2}, \\ \int_{0}^{T} e^{-\gamma t} u(T-t) \mathrm{d} t=e^{-\gamma T} M^{*}\left(y^{0}, \gamma\right), & \forall \gamma \in \Lambda_{3}, \\ \int_{0}^{T} t e^{-\gamma t} u(T-t) \mathrm{d} t=\frac{e^{-\gamma T}}{I(\gamma)} \widehat{M}\left(y^{0}, \gamma\right), & \forall \gamma \in \Lambda_{3} .\end{cases}
$$

Proposition 3.5 (gap-condition).

$$
\forall \alpha, \beta \in \sigma\left(L^{*}\right), \quad \text { with } \quad \alpha \neq \beta, \quad|\alpha-\beta|>\frac{1}{j_{0}^{2}} .
$$

Proof of Proposition 3.5. For all $\alpha, \beta \in \sigma\left(L^{*}\right)$, there exist $p, q \in \mathbb{N}^{*}$ where $p \neq q$, such that $j_{0}^{2} \alpha=p^{2}$ and $j_{0}^{2} \beta=q^{2}$ (see (3.1) and (3.3)), consequently

$$
|\alpha-\beta|=\frac{1}{j_{0}^{2}}\left|p^{2}-q^{2}\right| \geq \frac{1}{j_{0}^{2}}
$$

Thus, from the results in [13] (see Lemma 3.1. (b), page 1725-1726), we know that the sequence

$$
\left\{e^{-\alpha t}, t e^{-\alpha t}\right\}_{\alpha \in \sigma\left(L^{*}\right)}
$$

admits a biorthogonal family $\left\{q_{1, \alpha}, q_{2, \alpha}\right\}_{\alpha \in \sigma\left(L^{*}\right)}$ in $L^{2}(0, T)$, i.e., a family satisfying

$$
\left\{\begin{array} { l } 
{ \int _ { 0 } ^ { T } e ^ { - \alpha t } q _ { 1 , \beta } ( t ) \mathrm { d } t = \delta _ { \alpha \beta } , } \\
{ \int _ { 0 } ^ { T } t e ^ { - \alpha t } q _ { 1 , \beta } ( t ) \mathrm { d } t = 0 , }
\end{array} \quad \text { and } \quad \left\{\begin{array}{l}
\int_{0}^{T} e^{-\alpha t} q_{2, \beta}(t) \mathrm{d} t=0, \\
\int_{0}^{T} t e^{-\alpha t} q_{2, \beta}(t) \mathrm{d} t=\delta_{\alpha \beta},
\end{array}\right.\right.
$$

where $\alpha, \beta \in \sigma\left(L^{*}\right)$, which moreover satisfies that for every $\varepsilon>0$ there exists a constant $C_{\varepsilon, T}>0$ such that

$$
\left\|q_{j, \beta}\right\|_{L^{2}(0, T)} \leq C_{\varepsilon, T} e^{\varepsilon \beta}, \quad \beta \in \sigma\left(L^{*}\right), \quad j \in\{1,2\} .
$$

Using now the formulas in (3.17) and the property (3.18), we infer that an explicit formal solution $u$ is given by

$$
\begin{aligned}
u(T-t) & =\sum_{\alpha \in \Lambda_{1}} e^{-\alpha T} M\left(y^{0}, \alpha\right) q_{1, \alpha}(t)+\sum_{\beta \in \Lambda_{2}} \frac{e^{-\beta T} \widetilde{M}\left(y^{0}, \beta\right) q_{1, \beta}(t)}{I(\beta)} \\
& +\sum_{\gamma \in \Lambda_{3}} e^{-\gamma T}\left(M^{*}\left(y^{0}, \gamma\right) q_{1, \gamma}(t)+\frac{\widehat{M}\left(y^{0}, \gamma\right) q_{2, \gamma}(t)}{I(\gamma)}\right)
\end{aligned}
$$


Let us see that this series defines an element of $L^{2}(0, T)$ when $T>T_{0}$, i.e., the previous series converges in $L^{2}(0, T)$ if $T>T_{0}$. Indeed, from the definition of the minimal time $T_{0}$ (see (3.2)) and for any fixed $\varepsilon>0$, we can infer that there exists a positive constant $C_{\varepsilon}$ such that

$$
\frac{1}{|I(\zeta)|} \leq C_{\varepsilon} e^{\zeta\left(T_{0}+\varepsilon\right)}, \quad \forall \zeta \in \Lambda_{2} \cup \Lambda_{3}
$$

On one hand, we can use (3.18), (3.14) and (3.16), and get for any $\alpha \in \Lambda_{1}$ and $\gamma \in \Lambda_{3}$, a positive constant $C_{\varepsilon, T}>0$ for which

$$
\left\|e^{-\alpha T} M\left(y^{0}, \alpha\right) q_{1, \alpha}(t)\right\|_{L^{2}(0, T)}+\left\|e^{-\gamma T} M^{*}\left(y^{0}, \gamma\right) q_{1, \gamma}(t)\right\|_{L^{2}(0, T)} \leq C_{\varepsilon, T}\left\|y^{0}\right\|_{H_{0}^{1}}\left(e^{\alpha(\varepsilon-T)}+e^{\gamma(\varepsilon-T)}\right) .
$$

On the other hand, we can use (3.18), (3.15) and (3.16), and get for any $\beta \in \Lambda_{2}$ and $\gamma \in \Lambda_{3}$, a new positive constant $C_{\varepsilon, T}>0$ such that

$$
\begin{gathered}
\left\|\frac{e^{-\beta T}}{I(\beta)} \widetilde{M}\left(y^{0}, \beta\right) q_{2, \beta}(t)\right\|_{L^{2}} \leq C_{\varepsilon, T}\left\|y^{0}\right\|_{H_{0}^{1}} \frac{e^{\beta(\varepsilon-T)}}{|I(\beta)|} \leq C_{\varepsilon, T}\left\|y^{0}\right\|_{H_{0}^{1}} e^{\beta\left(T_{0}-T+2 \varepsilon\right)}, \\
\left\|\frac{e^{-\gamma T}}{I(\gamma)} \widehat{M}\left(y^{0}, \gamma\right) q_{2, \gamma}(t)\right\|_{L^{2}} \leq C_{\varepsilon, T}\left\|y^{0}\right\|_{H_{0}^{1}} \frac{e^{\gamma(\varepsilon-T)}}{|I(\gamma)|} \leq C_{\varepsilon, T}\left\|y^{0}\right\|_{H_{0}^{1}} e^{\gamma\left(T_{0}-T+2 \varepsilon\right)} .
\end{gathered}
$$

These previous two inequalities prove the absolute convergence of the series (3.19) which defines the control $u$ since $\varepsilon$ may be chosen arbitrarily small (take $\varepsilon \in\left(0, \frac{T-T_{0}}{2}\right)$ for instance). This proves the null controllability of system (1.1) at time $T$ when $T>T_{0}$.

\subsubsection{The negative null controllability result}

Let us prove that if $0<T<T_{0}$, then system (1.1) is not null controllable at time $T$. We argue by contradiction. Let us first remark that

$$
T_{0}=\max \left\{T_{0,1}, T_{0,2}\right\}
$$

with

$$
T_{0,1}=\limsup _{k \in \mathbb{N}^{*} \backslash i_{0} \mathbb{N}^{*}, k \rightarrow+\infty} \frac{\log \frac{1}{\left|I\left(k^{2}\right)\right|}}{k^{2}} \text { and } T_{0,2}=\limsup _{l \in \mathbb{N}^{*}, l \rightarrow+\infty} \frac{\log \frac{1}{\left|I\left(i_{0}^{2} l^{2}\right)\right|}}{i_{0}^{2} l^{2}}
$$

Assume that (1.1) is null-controllable at time $T<T_{0}$, system (1.1) is null-controllable at time $T$ if and only if there exists $C>0$ such that any solution $\theta$ of the adjoint problem (1.17) satisfies the observability inequality:

$$
\|\theta(0)\|_{H_{0}^{1}\left(0, \pi ; \mathbb{R}^{2}\right)}^{2} \leq C \int_{0}^{T}\left|B^{*} D \theta_{x}(0, t)\right|^{2} \mathrm{~d} t, \quad \forall \theta^{0} \in H_{0}^{1}\left(0, \pi ; \mathbb{R}^{2}\right)
$$


- Let us suppose $T_{0}=T_{0,1}$. Let us work with the particular solutions $\theta_{k}$ associated with initial data

$$
\theta_{k}^{0}=\frac{\widetilde{\Phi}_{1, k}^{*}}{\nu \widetilde{\psi}_{k}^{\prime}(0)} .
$$

With this choice, the solution $\theta_{k}$ of (1.17) is given by

$$
\theta_{k}(x, t)=\frac{\widetilde{\Phi}_{1, k}^{*}}{\nu \widetilde{\psi}_{k}^{\prime}(0)} e^{-k^{2}(T-t)}, \quad k \in \mathbb{N}^{*} \backslash i_{0} \mathbb{N}^{*} .
$$

From the definition of $T_{0,1}$ (see (3.20)), then there exists an sub-sequence $\left(k_{n}\right)_{n \geq 1} \subset \mathbb{N}^{*} \backslash i_{0} \mathbb{N}^{*}$ such that:

$$
T_{0,1}=\lim _{n \rightarrow+\infty} \frac{\log \frac{1}{\left|I\left(k_{n}^{2}\right)\right|}}{k_{n}^{2}} \in(0,+\infty]
$$

thus

$$
\begin{aligned}
\left\|\theta_{k_{n}}(\cdot, 0)\right\|_{H_{0}^{1}\left(0, \pi ; \mathbb{R}^{2}\right)}^{2} & =\frac{\left\|\widetilde{\Phi}_{1, k_{n}}^{*}\right\|_{H_{0}^{1}}^{2}}{\left|\nu \widetilde{\psi}_{k_{n}}^{\prime}(0)\right|^{2}} e^{-2 k_{n}^{2} T}=\frac{k_{n}^{2}+\left\|\widetilde{\psi}_{k_{n}}^{\prime}\right\|_{L^{2}}^{2}}{\left|\nu \widetilde{\psi}_{k_{n}}^{\prime}(0)\right|^{2}} e^{-2 k_{n}^{2} T}, \\
& \geq \frac{\pi}{2} \frac{\left|\sin \left(\frac{k_{n} \pi}{\sqrt{\nu}}\right)\right|^{2}}{\left|I\left(k_{n}^{2}\right)\right|} e^{-2 k_{n}^{2} T} \geq k_{n}^{2} \frac{\pi}{2} \frac{4}{i_{0}^{2}} \frac{1}{\left|I\left(k_{n}^{2}\right)\right|^{2}} e^{-2 k_{n}^{2} T}, \\
& \geq k_{n}^{2} \frac{\pi}{2} \frac{4}{i_{0}^{2}} e^{-2 k_{n}^{2}\left[T-\frac{\log \left|I\left(k_{n}^{2}\right)\right|}{k_{n}^{2}}\right]}, \quad \\
& \geq k_{n}^{2} \frac{\pi}{2} \frac{4}{i_{0}^{2}} e^{-2 k_{n}^{2}\left[T-T_{0,1}+\varepsilon\right]} \underset{n \rightarrow+\infty}{\longrightarrow}+\infty, \quad \varepsilon \in\left(0, T_{0,1}-T\right),
\end{aligned}
$$

and

$$
\int_{0}^{T}\left|B^{*} D \frac{\partial \theta_{k_{n}}(0, t)}{\partial x}(0, t)\right|^{2} \mathrm{~d} t=\int_{0}^{T} e^{-2 k_{n}^{2} t} \mathrm{~d} t \underset{n \rightarrow+\infty}{\longrightarrow} 0
$$

This proves that the observability inequality (3.21) does not hold.

- Let us suppose now, $T_{0}=T_{0,2}$. Let us work with the particular solutions $\theta^{l}$ associated with initial data

$$
\theta^{0}=a_{l} \widehat{\Phi}_{1, l}^{*}+b_{l} \Phi_{2, j_{0} l}^{*}, \quad l \geq 1,
$$

with $a_{l}, b_{l} \in \mathbb{R}$, to be determined. Recall that

$$
\int_{0}^{\pi} \widehat{\psi}_{l}(x) \varphi_{j_{0} l}(x) \mathrm{d} x=0 \quad(\text { see }(3.5))
$$

With this choice, the solution $\theta^{l}$ of (1.17) is given by

$$
\theta^{l}(\cdot, t)=a_{l} e^{-i_{0}^{2} l^{2}(T-t)}\left[\widehat{\Phi}_{1, l}+(T-t) I\left(i_{0}^{2} l^{2}\right) \Phi_{2, j_{0} l}^{*}\right]+b_{l} e^{-i_{0}^{2} l^{2}(T-t)} \Phi_{2, j_{0} l}^{*}, \quad \forall l \geq 1,
$$


thus, the observability inequality (3.21) becomes

$$
A_{1, l} \leq C A_{2, l}
$$

with, for all $l \geq 1$

$$
\left\{\begin{array}{l}
A_{1, l}=e^{-2 i_{0}^{2} l^{2} T}\left\{i_{0}^{2} l^{2}\left|a_{l}\right|^{2}+\left[\left|a_{l}\right|^{2}\left\|\psi_{l}^{\prime}\right\|_{L^{2}(0, \pi)}^{2}+i_{0}^{2} l^{2}\left(b_{l}+T a_{l} I\left(i_{0}^{2} l^{2}\right)\right)^{2}\right]\right\} \geq e^{-2 i_{0}^{2} l^{2} T}\left|a_{l}\right|^{2}, \\
A_{2, l}=\int_{0}^{T} e^{-2 i_{0}^{2} l^{2} t}\left|a_{l} \psi_{l}^{\prime}(0)+\left(b_{l}+t a_{l} I\left(i_{0}^{2} l^{2}\right)\right) \varphi_{j_{0} l}^{\prime}(0)\right|^{2} \mathrm{~d} t .
\end{array}\right.
$$

We can choose $a_{l}=I\left(i_{0}^{2} l^{2}\right)$ and $b_{l}=-I\left(i_{0}^{2} l^{2}\right) \psi_{l}^{\prime}(0) / \varphi_{j_{0} l}^{\prime}(0)$, we obtain for a new constant $C>0$ not depending on $l$, such that:

$$
\frac{A_{1, l}}{A_{2, l}} \geq \frac{e^{-2 i_{0}^{2} l^{2} T}}{\left|I\left(i_{0}^{2} l^{2}\right) \varphi_{j_{0} l}^{\prime}(0)\right|^{2} \int_{0}^{T} t^{2} e^{-2 i_{0}^{2} l^{2} t} \mathrm{~d} t} \geq \frac{e^{-2 i_{0}^{2} l^{2} T}}{\left|I\left(i_{0}^{2} l^{2}\right)\right|^{2}}, \quad \forall l \geq 1
$$

From the definition of $T_{0,2}$ (see (3.20)), there exists a sub-sequence $\left\{l_{n}\right\}_{n \geq 1}$ such that:

$$
T_{0,2}=\lim _{n \rightarrow+\infty} \frac{\log \frac{1}{\left|I\left(i_{0}^{2} l_{n}^{2}\right)\right|}}{i_{0}^{2} l_{n}^{2}} \in(0,+\infty] .
$$

Assume $0<T_{0,2}<+\infty$. In this case, for every $\varepsilon>0$, there exits a positive integer $n_{\varepsilon} \geq 1$ such that

$$
\frac{1}{\left|I\left(i_{0}^{2} l_{n}^{2}\right)\right|}>e^{i_{0}^{2} l_{n}^{2}\left(T_{0}^{2}-\varepsilon\right)}, \quad \forall n \geq n_{\varepsilon}
$$

thus, $\forall n \geq n_{\varepsilon}$

$$
\frac{A_{1, l_{n}}}{A_{2, l_{n}}} \geq \frac{e^{-2 i_{0}^{2} l_{n}^{2} T}}{\left|I\left(i_{0}^{2} l_{n}^{2}\right)\right|^{2}} \geq e^{2 i_{0}^{2} l_{n}^{2}\left(T_{0,2}-T-\varepsilon\right)}
$$

Taking for instance $\varepsilon=\frac{T_{0,2}-T}{2}$, we have

$$
e^{2 i_{0}^{2} l_{n}^{2}\left(T_{0,2}-T-\varepsilon\right)}=e^{i_{0}^{2} l_{n}^{2}\left(T_{0,2}-T\right)} \underset{n \rightarrow+\infty}{\longrightarrow}+\infty .
$$

This proves that the observability inequality (3.21) does not hold and finishes the proof of the negative null controllability result of (1.1).

\section{Appendix A. Reformulation of minimal time in Theorem 1.1}

Let us begin this section by reformulating the minimal times introduced in Theorem 1.1. Let us first recall that

$$
B^{*} D \frac{\partial \Phi_{1, k}^{*}}{\partial x}(0)=\psi_{k}^{\prime}(0)=-\frac{\int_{0}^{\pi} q(s) \varphi_{k}(s) \sin \left(\frac{k}{\sqrt{\nu}}(\pi-s)\right) \mathrm{d} s}{\nu \sin \left(\frac{k \pi}{\sqrt{\nu}}\right)} .
$$


In all the sequel, assume that condition (1.11) is holds. We have

Proposition A.1. Let us consider the definitions of the Theorem 1.1. One has

1.

$$
T_{1}=\limsup _{k \rightarrow+\infty}\left\{\frac{1}{k^{2}} \log \frac{\left|\sin \left(\frac{k \pi}{\sqrt{\nu}}\right)\right|}{\left|\int_{0}^{\pi} q(s) \varphi_{k}(s) \sin \left(\frac{k}{\sqrt{\nu}}(\pi-s)\right) \mathrm{d} s\right|}\right\} \in[0,+\infty] .
$$

If $\sqrt{\nu}>1$, then

$$
\widetilde{T}_{2}=\limsup _{k \rightarrow+\infty}\left\{\frac{1}{i_{k}^{2}} \log \frac{1}{\left|\int_{0}^{\pi} q(s) \varphi_{i_{k}}(s) \sin \left(\frac{i_{k}}{\sqrt{\nu}}(\pi-s)\right) \mathrm{d} s\right|}\right\} \in[0,+\infty]
$$

2. If $\sqrt{\nu}<1$, then

$$
\widehat{T}_{2}=\limsup _{k \rightarrow+\infty}\left\{\frac{1}{k^{2}} \log \frac{1}{\left|\int_{0}^{\pi} q(s) \varphi_{k}(s) \sin \left(\frac{k}{\sqrt{\nu}}(\pi-s)\right) \mathrm{d} s\right|}\right\} \geq T_{1},
$$

consequently

$$
\widehat{T}_{0}=\widehat{T}_{2}
$$

Poof of Proposition A.1. Let us recall that for all $k \geq 1$, one has

$$
\Phi_{1, k}^{*}:=\left(\begin{array}{l}
\varphi_{k} \\
\psi_{k}
\end{array}\right), \Phi_{2, k}^{*}:=\left(\begin{array}{l}
0 \\
\varphi_{k}
\end{array}\right) \quad(\text { see }(1.7))
$$

and

$$
\psi_{1, k}:=\frac{\Phi_{1, k}^{*}}{B^{*} D \frac{\partial \Phi_{1, k}^{*}}{\partial x}(0)}=\frac{\Phi_{1, k}^{*}}{\nu \psi_{k}^{\prime}(0)} \quad(\text { see }(1.9)),
$$

where (see (2.1))

$$
\psi_{k}(x)=\psi_{k}^{\prime}(0) \frac{\sqrt{\nu}}{k} \sin \left(\frac{k x}{\sqrt{\nu}}\right)+\frac{\sqrt{\nu}}{\nu k} \int_{0}^{x} \sin \left(\frac{k}{\sqrt{\nu}}(x-\xi)\right) q(\xi) \varphi_{k}(\xi) \mathrm{d} \xi
$$

Moreover, one has

$$
\psi_{k}^{\prime}(x)=\psi_{k}^{\prime}(0) \cos \left(\frac{k x}{\sqrt{\nu}}\right)+\frac{1}{\nu} \int_{0}^{x} \cos \left(\frac{k}{\sqrt{\nu}}(x-\xi)\right) q(\xi) \varphi_{k}(\xi) \mathrm{d} \xi
$$

We immediately see that there is a constant $C>0$ such that

$$
\left\|\psi_{k}^{\prime}\right\|_{L^{2}(0, \pi)}^{2} \leq C\left|\nu \psi_{k}^{\prime}(0)\right|^{2}+C .
$$


1. On one hand, using the previous inequality (A.2), one has

$$
\frac{k^{2}}{\left|\nu \psi_{k}^{\prime}(0)\right|^{2}} \leq\left\|\psi_{1, k}\right\|_{H_{0}^{1}}^{2}=\frac{k^{2}+\left\|\psi_{k}^{\prime}\right\|_{L^{2}}^{2}}{\left|\nu \psi_{k}^{\prime}(0)\right|^{2}} \leq \frac{k^{2}+C\left|\nu \psi_{k}^{\prime}(0)\right|^{2}+C}{\left|\nu \psi_{k}^{\prime}(0)\right|^{2}} \leq \frac{k^{2}+C}{\left|\nu \psi_{k}^{\prime}(0)\right|^{2}}+C,
$$

thus

$$
\begin{aligned}
T_{1} & =\limsup _{k \rightarrow+\infty} \frac{\log \left\|\psi_{1, k}\right\|_{H_{0}^{1}}}{k^{2}}=\limsup _{k \rightarrow+\infty} \frac{-\log \left|\nu \psi_{k}^{\prime}(0)\right|}{k^{2}} \\
& =\limsup _{k \rightarrow+\infty}\left\{\frac{1}{k^{2}} \log \frac{\left|\sin \left(\frac{k \pi}{\sqrt{\nu}}\right)\right|}{\left|\int_{0}^{\pi} q(s) \varphi_{k}(s) \sin \left(\frac{k}{\sqrt{\nu}}(\pi-s)\right) \mathrm{d} s\right|}\right\} \quad \text { (see (A.1)). }
\end{aligned}
$$

On the other hand, for all $k \geq 1$ and $\sqrt{\nu}>1$, one has

$$
\left|i_{k}-\sqrt{\nu} k\right|<\frac{1}{2}
$$

where $i_{k}$ is the nearest integer to $\sqrt{\nu} k$, thus $\left|\frac{i_{k}}{\sqrt{\nu}}-k\right|<\frac{1}{2 \sqrt{\nu}}<\frac{1}{2}$. Consequently

$$
2\left|\frac{i_{k}}{\sqrt{\nu}}-k\right| \leq\left|\sin \left(\frac{i_{k} \pi}{\sqrt{\nu}}\right)\right| \leq \pi\left|\frac{i_{k}}{\sqrt{\nu}}-k\right| .
$$

Thus

$$
\begin{aligned}
& \frac{\left\|\psi_{1, i_{k}}-\psi_{2, k}\right\|_{H_{0}^{1}}^{2}}{\left|i_{k}^{2}-\nu k^{2}\right|^{2}}=\frac{1}{\left|i_{k}^{2}-\nu k^{2}\right|^{2}}\left(\frac{i_{k}^{2}}{\left|\nu \psi_{i_{k}}^{\prime}(0)\right|^{2}}+\frac{\left\|\nu \varphi_{k}^{\prime}(0) \psi_{i_{k}}^{\prime}-\nu \psi_{i_{k}}^{\prime}(0) \varphi_{k}^{\prime}\right\|_{L^{2}}^{2}}{\left|\nu \psi_{i_{k}}^{\prime}(0)\right|^{2}\left|\nu \varphi_{k}^{\prime}(0)\right|^{2}}\right) \\
& \leq \frac{1}{\left|i_{k}^{2}-\nu k^{2}\right|^{2}}\left(\frac{i_{k}^{2}}{\left|\nu \psi_{i_{k}}^{\prime}(0)\right|^{2}}+\frac{2\left\|\psi_{i_{k}}^{\prime}\right\|_{L^{2}}^{2}}{\left|\nu \psi_{i_{k}}^{\prime}(0)\right|^{2}}+\frac{2 k^{2}}{\left|\nu \varphi_{k}^{\prime}(0)\right|^{2}}\right) \\
& \leq \frac{1}{\left|i_{k}^{2}-\nu k^{2}\right|^{2}}\left(\frac{i_{k}^{2}+C}{\left|\nu \psi_{i_{k}}^{\prime}(0)\right|^{2}}+C+\frac{\pi}{\nu}\right) \\
& \leq \frac{C^{\prime} i_{k}^{2}}{\left|i_{k}^{2}-\nu k^{2}\right|^{2}}\left(\frac{\left|\sin \left(\frac{i_{k} \pi}{\sqrt{\nu}}\right)\right|^{2}}{\left|\int_{0}^{\pi} q(s) \varphi_{i_{k}}(s) \sin \left(\frac{i_{k}}{\sqrt{\nu}}(\pi-s)\right) \mathrm{d} s\right|^{2}}\right) \\
& \leq \frac{C^{\prime} i_{k}^{2}}{\left|i_{k}+\sqrt{\nu} k\right|^{2}} \frac{\pi^{2}}{\nu}\left(\frac{1}{\left|\int_{0}^{\pi} q(s) \varphi_{i_{k}}(s) \sin \left(\frac{i_{k}}{\sqrt{\nu}}(\pi-s)\right) \mathrm{d} s\right|^{2}}\right)
\end{aligned}
$$


and

$$
\begin{aligned}
\frac{\left\|\psi_{1, i_{k}}-\psi_{2, k}\right\|_{H_{0}^{1}}^{2}}{\left|i_{k}^{2}-\nu k^{2}\right|^{2}} & =\frac{1}{\left|i_{k}^{2}-\nu k^{2}\right|^{2}}\left(\frac{i_{k}^{2}}{\left|\nu \psi_{i_{k}}^{\prime}(0)\right|^{2}}+\frac{\left\|\nu \varphi_{k}^{\prime}(0) \psi_{i_{k}}^{\prime}-\nu \psi_{i_{k}}^{\prime}(0) \varphi_{k}^{\prime}\right\|_{L^{2}}^{2}}{\left|\nu \psi_{i_{k}}^{\prime}(0)\right|^{2}\left|\nu \varphi_{k}^{\prime}(0)\right|^{2}}\right) \\
& \left.\geq \frac{1}{\left|i_{k}^{2}-\nu k^{2}\right|^{2}} \frac{\left|\sin \left(\frac{i_{k} \pi}{\sqrt{\nu}}\right)\right|^{2}}{\left|\nu \psi_{i_{k}}^{\prime}(0)\right|^{2}}\right) \\
& \geq \frac{i_{k}^{2}}{\left|i_{k}^{2}-\nu k^{2}\right|^{2}}\left(\frac{1}{\left|\int_{0}^{\pi} q(s) \varphi_{i_{k}}(s) \sin \left(\frac{i_{k}}{\sqrt{\nu}}(\pi-s)\right) \mathrm{d} s\right|^{2}}\right) \\
& \geq \frac{i_{k}^{2}}{\left|i_{k}+\sqrt{\nu}\right|^{2}} \frac{4}{\nu}\left(\frac{1}{\left|\int_{0}^{\pi} q(s) \varphi_{i_{k}}(s) \sin \left(\frac{i_{k}}{\sqrt{\nu}}(\pi-s)\right) \mathrm{d} s\right|^{2}}\right)
\end{aligned}
$$

and then, we deduce that

$$
\widetilde{T}_{2}=\limsup _{k \rightarrow+\infty} \frac{-\log \left|\int_{0}^{\pi} q(s) \varphi_{i_{k}}(s) \sin \left(\frac{i_{k}}{\sqrt{\nu}}(\pi-s)\right) \mathrm{d} s\right|}{\nu k^{2}} .
$$

2. Similarly, we show that, for $\sqrt{\nu}<1$

$$
\widehat{T}_{0}=\widehat{T}_{2}=\limsup _{k \rightarrow+\infty} \frac{-\log \left|\int_{0}^{\pi} q(s) \varphi_{k}(s) \sin \left(\frac{k}{\sqrt{\nu}}(\pi-s)\right) \mathrm{d} s\right|}{k^{2}} \geq T_{1} .
$$

In the following Proposition A.2 we will give a positive answer to the natural question: Let $\tau_{0} \in[0,+\infty]$, is there $\sqrt{\nu} \notin \mathbb{Q}^{*}$ and $q \in L^{\infty}(0, \pi)$ such that $\widetilde{T}_{0}=\tau_{0}\left(\right.$ or $\left.\widehat{T}_{0}=\tau_{0}\right)$ ?

Proposition A.2. Let $\sqrt{\nu} \notin \mathbb{Q}$.

1. For any $\tau_{0} \in[0,+\infty]$, there exists $\sqrt{\nu}>1$ and $q \in L^{\infty}(0, \pi)$ such that

$$
\widetilde{T}_{0}=\max \left\{T_{1}, \widetilde{T}_{2}\right\}=\tau_{0}
$$

2. For any $\tau_{0} \in[0,+\infty]$, there exists $\sqrt{\nu}<1$ and $q \in L^{\infty}(0, \pi)$ such that

$$
\widehat{T}_{0}=\widehat{T}_{2}=\tau_{0} .
$$

Proof of Proposition A.2 In the sequel, we will work with $\widetilde{T}_{0}$. The arguments to prove that $\widehat{T}_{0}=\tau_{0} \in[0,+\infty]$, are similar. Let us recall

Lemma A.3. 1. Let us fixed $\tau_{0} \in(0, \infty), x_{0} \in[0, \infty)$ and $\varepsilon>0$. Then, there exist an irrational number $\nu>0$ and a sequence of rational numbers $\left\{p_{k} / q_{k}\right\}_{k \geq 1}$ such that $p_{k}$ and $q_{k}$ are co-prime positive integers, the sequences $\left\{p_{k}\right\}_{k \geq 1}$ and $\left\{q_{k}\right\}_{k \geq 1}$ are strictly increasing,

$$
\left|\nu-x_{0}\right| \leq \varepsilon \quad \text { and } \quad \lim _{k \rightarrow \infty} e^{\tau_{0} q_{k}^{2}}\left|\nu-\frac{p_{k}}{q_{k}}\right|=1 .
$$


Moreover, for any $k \geq 1$ one has

$$
0<\left|q_{k} \nu-p_{k}\right| \leq|q \nu-p|, \quad \forall p, q \in \mathbb{N}^{*}, \quad \text { with } q<q_{k+1} .
$$

2. For any $\sigma \in(0, \infty), x_{0} \in[0, \infty)$ and $\varepsilon>0$, there exists an irrational number $\nu>0$ and a sequence of rational numbers $\left\{p_{k} / q_{k}\right\}_{k \geq 1}$ such that $p_{k}$ and $q_{k}$ are co-prime positive integers, the sequences $\left\{p_{k}\right\}_{k \geq 1}$ and $\left\{q_{k}\right\}_{k \geq 1}$ are strictly increasing and

$$
\left|\nu-x_{0}\right| \leq \varepsilon \quad \text { and } \quad \lim _{k \rightarrow \infty} e^{q_{k}^{2+\sigma}}\left|\nu-\frac{p_{k}}{q_{k}}\right|=0
$$

Proof of Lemma A.3 See for example [3] Lemma A.1, page 35.

Note that by choosing $q(x)=1, x \in[0, \pi]$, we obtain

$$
\frac{\left|\sin \left(\frac{k \pi}{\sqrt{\nu}}\right)\right|}{\left|\int_{0}^{\pi} q(s) \varphi_{k}(s) \sin \left(\frac{k}{\sqrt{\nu}}(\pi-s)\right) \mathrm{d} s\right|}=\frac{\left|\sin \left(\frac{k \pi}{\sqrt{\nu}}\right)\right|}{\left|\int_{0}^{\pi} \varphi_{k}(s) \sin \left(\frac{k}{\sqrt{\nu}}(\pi-s)\right) \mathrm{d} s\right|}=\sqrt{\frac{\pi}{2}} \frac{k|\nu-1|}{\nu},
$$

and thus $T_{1}=0$. But

$$
\frac{1}{\left|\int_{0}^{\pi} q(s) \varphi_{i_{k}}(s) \sin \left(\frac{i_{k}}{\sqrt{\nu}}(\pi-s)\right) \mathrm{d} s\right|}=\frac{1}{\left|\int_{0}^{\pi} \varphi_{i_{k}}(s) \sin \left(\frac{i_{k}}{\sqrt{\nu}}(\pi-s)\right) \mathrm{d} s\right|}=\sqrt{\frac{\pi}{2}} \frac{i_{k}|\nu-1|}{\nu\left|\sin \left(\frac{i_{k} \pi}{\sqrt{\nu}}\right)\right|},
$$

thus in this case

$$
\widetilde{T}_{0}=\widetilde{T}_{2}=\limsup _{k \rightarrow+\infty} \frac{-\log \left|\sin \left(\frac{i_{k} \pi}{\sqrt{\nu}}\right)\right|}{i_{k}^{2}}
$$

Finally, we have

Lemma A.4. Let $\nu \notin \mathbb{Q}^{*}$ and $i_{k}$ nearest integer to $\sqrt{\nu} k$, for each $k \geq 1$. If $\nu>1$ then

$$
\limsup _{k \rightarrow+\infty} \frac{-\log \left|\sin \left(\frac{i_{k} \pi}{\sqrt{\nu}}\right)\right|}{i_{k}^{2}}=\limsup _{k \rightarrow+\infty} \frac{-\log |\sin (\sqrt{\nu} k \pi)|}{\nu k^{2}} .
$$

Proof of Lemma A.4 We have

$$
\begin{aligned}
\limsup _{k \rightarrow+\infty} \frac{-\log \left|\sin \left(\frac{i_{k} \pi}{\sqrt{\nu}}\right)\right|}{i_{k}^{2}} & =\limsup _{k \rightarrow+\infty} \frac{-\log \left|\frac{i_{k}}{\sqrt{\nu}}-k\right|}{\nu k^{2}}, \quad\left(\operatorname{see}(A .4), \text { and } \lim \frac{i_{k}^{2}}{\nu k^{2}}=1\right) \\
& =\limsup _{k \rightarrow+\infty} \frac{-\log \left|\sqrt{\nu} k-i_{k}\right|}{\nu k^{2}}, \\
& =\limsup _{k \rightarrow+\infty} \frac{-\log |\sin (\sqrt{\nu} k)|}{\nu k^{2}} \quad(\operatorname{see}(A .3)) .
\end{aligned}
$$


Let us fixed $x_{0} \geq 0$ et $\varepsilon>0$. We will distinguish three different cases in the proof of Proposition A.2. Case $\tau_{0}=0$ : Given $x_{0} \geq 1$ and $\varepsilon>0$ such that $x_{0}-\varepsilon>1$. Taking $\nu>1$ a irrational algebraic number of order $n \geq 2$, we deduce the existence of a positive constant $C>0$ such that

$$
\mid \sin \left(i_{k} \pi / \sqrt{\nu} \mid \geq \frac{C}{i_{k}^{n-1}}, \quad \forall k \geq 1\right.
$$

Consequently $\widetilde{T}_{0}=\widetilde{T}_{2}=0$.

Case $\tau_{\mathbf{0}} \in(\mathbf{0}, \infty)$ : Given $\tau_{0}, x_{0} \geq 0$ and $\varepsilon>0$ such that $x_{0}-\varepsilon>1$. We deduce the existence of a irrational algebraic number $\nu>1$ which fulfills (A.5) and (A.6) for the sequences of positive integers $\left\{p_{k}\right\}_{k \geq 0}$ et $\left\{q_{k}\right\}_{k \geq 0}$. From (A.5) we deduce $\lim \left(p_{k}-q_{k} \sqrt{\nu}\right)=0, \lim p_{k} / q_{k}=\sqrt{\nu}$. Thus from the Lemma A.4, we have

$$
\begin{aligned}
\widetilde{T}_{2}=\limsup _{k \rightarrow+\infty} \frac{-\log \left|\sin \left(\frac{i_{k} \pi}{\sqrt{\nu}}\right)\right|}{i_{k}^{2}} & =\limsup _{k \rightarrow+\infty} \frac{-\log |\sin (\sqrt{\nu} k \pi)|}{\nu k^{2}}, \\
& =\limsup _{k \rightarrow+\infty} \frac{-\log |\sin (\sqrt{\nu} k \pi-h \pi)|}{\nu k^{2}}, \quad \forall h \in \mathbb{N}, \\
& \geq \limsup _{k \rightarrow+\infty} \frac{-\log \left|\sin \left(\sqrt{\nu} q_{k} \pi-h \pi\right)\right|}{\nu q_{k}^{2}}, \\
& =\limsup _{k \rightarrow+\infty} \frac{-\log \left|\sin \pi\left(\sqrt{\nu} q_{k}-p_{k}\right)\right|}{\nu q_{k}^{2}}, \\
& =\limsup _{k \rightarrow+\infty} \frac{-\log \left|\left(\pi\left(\sqrt{\nu} q_{k}-p_{k}\right)\right)\right|}{\nu q_{k}^{2}}, \\
& =\lim _{k \rightarrow+\infty} \frac{-\log \left|\pi q_{k} e^{-\tau_{0} q_{k}^{2}}\right|}{\nu q_{k}^{2}}=\frac{\tau_{0}}{\nu} .
\end{aligned}
$$

Let us now show $\widetilde{T}_{2} \leq \tau_{0}$. Note that we can deduce from the previous result that

$$
\limsup _{k \rightarrow+\infty} \frac{-\log \left|\sin \pi\left(\sqrt{\nu} q_{k}-p_{k}\right)\right|}{\nu q_{k}^{2}}=\frac{\tau_{0}}{\nu}
$$

thus, there exists $k_{0}(\varepsilon) \geq 1$ such that

$$
\frac{-\log \left|\sin \pi\left(\sqrt{\nu} q_{k}-p_{k}\right)\right|}{\nu q_{k}^{2}} \leq \frac{\tau_{0}}{\nu}+\varepsilon, \quad \forall k \geq k_{0}(\varepsilon)
$$

for every $n \geq 1$, there is $i_{n} \in \mathbb{N}^{*}$ for which

$$
\left|\sqrt{\nu} n-i_{n}\right|<\frac{1}{2}, \quad \forall n \geq 1
$$

Let us take $n_{0}(\varepsilon)=q_{k_{0}(\varepsilon)} \geq 1$. Thus, using that the sequence $\left\{q_{k}\right\}_{k \geq 1}$ is strictly increasing, if $n \geq n_{0}(\varepsilon)$, we infer the existence of $k \geq k_{0}(\varepsilon)$ such that $q_{k} \leq n<q_{k+1}$. These last properties together with the second formula 
in (A.6) allow us to write

$$
\left|\sqrt{\nu} q_{k}-p_{k}\right| \leq\left|\sqrt{\nu} n-i_{n}\right|<\frac{1}{2}
$$

and

$$
\begin{aligned}
|\sin (\sqrt{\nu} n \pi)| & =\left|\sin \left(\pi\left(\sqrt{\nu} n-i_{n}\right)\right)\right| \\
& =\sin \left(\pi\left|\sqrt{\nu} n-i_{n}\right|\right) \\
& \geq \sin \left(\pi\left|\sqrt{\nu} q_{k}-p_{k}\right|\right) \\
& =\left|\sin \left(\pi\left(\sqrt{\nu} q_{k}-p_{k}\right)\right)\right|, \quad \forall n \geq n_{0}(\varepsilon), \text { such that } q_{k} \leq n<q_{k+1} .
\end{aligned}
$$

Since $q_{k} \leq n<q_{k+1}$, the previous inequality and (A.8) give

$$
\frac{-\log |\sin (\sqrt{\nu} n \pi)|}{\nu n^{2}} \leq \frac{-\log \left|\sin \left(\pi\left(\sqrt{\nu} q_{k}-p_{k}\right)\right)\right|}{\nu q_{k}^{2}} \leq \frac{\tau_{0}}{\nu}+\varepsilon, \quad \forall n \geq n_{0}(\varepsilon), \text { such that } q_{k} \leq n<q_{k+1},
$$

and

$$
\widetilde{T}_{2}=\limsup \frac{-\log |\sin (\sqrt{\nu} n \pi)|}{\nu n^{2}} \leq \frac{\tau_{0}}{\nu}+\varepsilon, \quad \forall \varepsilon>0
$$

Case $\tau_{0}=\infty$ : Fix $\sigma>0$ and take $x_{0} \in(0,+\infty)$ and $\varepsilon>0$ such that $x_{0}-\varepsilon>1$. We apply the second item in Lemma A.3 with, for instance, $\sigma=1 / 2$. We deduce the existence of a positive irrational number $\nu>1$ and two sequences of positive integers $\left\{p_{k}\right\}_{k \geq 0}$ and $\left\{q_{k}\right\}_{k \geq 0}$ which fulfills (A.7). Thus, we deduce

$$
\begin{aligned}
\widetilde{T}_{2} & =\limsup _{k \rightarrow+\infty} \frac{-\log |\sin (\sqrt{\nu} k \pi)|}{\nu k^{2}} \\
& \geq \limsup _{k \rightarrow+\infty} \frac{-\log \left|\sin \left(\sqrt{\nu} q_{k} \pi\right)\right|}{\nu q_{k}^{2}} \\
& =\limsup _{k \rightarrow+\infty} \frac{-\log \left|\sin \left(\pi\left(q_{k} \sqrt{\nu}-p_{k}\right)\right)\right|}{\nu q_{k}^{2}} \\
& =\limsup _{k \rightarrow+\infty} \frac{-\log \left|\pi\left(q_{k} \sqrt{\nu}-p_{k}\right)\right|}{\nu q_{k}^{2}} \\
& =\lim _{k \rightarrow+\infty} \frac{-\log \left|\pi q_{k} e^{-q_{k}^{2+\sigma}}\right|}{\nu q_{k}^{2}}=\infty .
\end{aligned}
$$

This shows that $\widetilde{T}_{2}=\infty$ and finishes the third case and the proof of Proposition A.2.

\section{REFERENCES}

[1] F. Ammar Khodja, A. Benabdallah, M. Gonzàlez-Burgos and L. Teresa, The Kalman condition for the boundary controllability of coupled parabolic systems. Bounds on biorthogonal families to complex matrix exponentials. J. Math. Pures Appl. 96 (2011) 505-590.

[2] F. Ammar Khodja, A. Benabdallah, M. Gonzàlez-Burgos and L. Teresa, Minimal time for the null controllability of parabolic systems: the effect of the condensation index of complex sequences. J. Funct. Anal. 267 (2014) 2077-2151.

[3] F. Ammar Khodja, A. Benabdallah, M. Gonzàlez-Burgos and L. Teresa, New phenomena for the null controllability of parabolic systems: Minimal time and geometrical dependence. J. Math. Anal. Appl. 444 (2016) 1071-1113.

[4] F. Ammar Khodja, A. Benabdallah, M. Gonzàlez-Burgos and M. Morancey, Quantitative Fattorini-Hautus test and minimal null control time for parabolic problems. J. Math. Pures Appl. 122 (2019) 198-234. 
[5] A. Benabdallah, F. Boyer and M. Morancey, A block moments method to handle spectral condensation phenomenon in parabolic control problems. Preprint (2018). Available at https://hal.archives-ouvertes.fr/hal-01949391.

[6] V. Bernstein, Lecons sur les Progrès Récents de la Théorie des Séries de Dirichlet. Gauthier-Villars (1933).

[7] O. Christensen, An Introduction to Frames and Riesz Bases, Applied and Numerical Harmonic Analysis. Birkhauser Basel (2003).

[8] S. Dolecki, Observability for the one-dimensional heat equation. Studia Math. 48 (1973) 291-305.

[9] M. Duprez, Controllability of a $2 \times 2$ parabolic system by one force with space-dependent coupling term of order one. ESAIM: COCV 23 (2017) 1473-1498.

[10] H.O. Fattorini, Some remarks on complete controllability. SIAM J. Control 4 (1966) 686-694.

[11] H.O. Fattorini and D.L. Russell, Exact controllability theorems for linear parabolic equations in one space dimension. Arch. Rational Mech. Anal. 43 (1971) 272-292.

[12] H.O. Fattorini and D.L. Russell, Uniform bounds on biorthogonal functions for real exponentials with an application to the control theory of parabolic equations. Quart. Appl. Math. 32 (1974/75) 45-69.

[13] E. Fernàndez-Cara, M. Gonzàlez-Burgos and L. de Teresa, Boundary controllability of parabolic coupled equations. J. Funct. Anal. 259 (2010) 1720-1758.

[14] A.V. Fursikov and O.Y. Imanuvilov. Controllability of evolution equations. Vol. 34 of Lecture Notes Series. Seoul National University Research. Institute of Mathematics Global Analysis Research Center, Seoul (1996).

[15] J.-L. Lions, Contrôle optimal de systèmes gouvernés par des équations aux dérivées partielles. Avant propos de P. Lelong. Paris: Dunod (1968).

[16] G. Lebeau and L. Robbiano. Contrôle exact de l'équation de la chaleur. Commun. Partial Differ. Equ. 20 (1995) $335-356$.

[17] G. Olive, Boundary approximate controllability of some linear parabolic systems. Evol. Equ. Control Theory 3 (2014) 167-189.

[18] L. Schwartz, Etude des Sommes d'Exponentielles, 2ème éd., Publications de l'Institut de Mathématiques de l'Université de Strasbourg, V. Actualités Sci. Ind., Hermann, Paris (1959).

[19] J.R. Shackell, Overconvergence of Dirichlet series with complex exponents. J. Anal. Math. 22 (1969) $135-170$.

[20] M. Tucsnak and G. Weiss, Observation and Control for Operator Semigroups. Birkhauser Advanced Texts: Basler Lehrbucher, Birkhauser Verlag, Basel (2009). 